\title{
Differences in coding provided by proprioceptive and vestibular sensory signals may contribute to lateral instability in vestibular loss subjects
}

\author{
John H. J. Allum • Lars B. Oude Nijhuis • \\ Mark G. Carpenter
}

Received: 16 March 2006 / Accepted: 16 August 2007 / Published online: 12 September 2007

(C) Springer-Verlag 2007

\begin{abstract}
One of the signatures of balance deficits observed in vestibular loss subjects is the greater instability in the roll compared to pitch planes. Directional differences in the timing and strengths of vestibular and proprioceptive sensory signals between roll and pitch may lead to a greater miscalculation of roll than pitch motion of the body in space when vestibular input is absent. For this reason, we compared the timing and amplitude of vestibular information, (observable in stimulus-induced head accelerations when subjects are tilted in different directions), with that of proprioceptive information caused by stimulus induced rotations of ankle and hip joints [observable as short latency (SL) stretch responses in leg and trunk muscle EMG activity]. We attempted to link the possible mode of sensory interaction with the deficits in balance control. Six subjects with bilaterally absent vestibular function and 12 age-matched controls were perturbed, while standing, in 8 directions of pitch and roll support surface rotation in random order. Body segment movements were recorded with a
\end{abstract}

J. H. J. Allum · L. B. Oude Nijhuis · M. G. Carpenter

Department of ORL, University Hospital,

Basel, Switzerland

L. B. Oude Nijhuis

Department of Neurology,

Radboud University Medical Centre,

Nijmegen, The Netherlands

\section{G. Carpenter}

School of Human Kinetics,

University of British Columbia,

Vancouver, Canada

J. H. J. Allum ( $\square)$

University ORL Clinic, Petersgraben 4,

4031 Basel, Switzerland

e-mail: jallum@uhbs.ch motion analysis system, head accelerations with accelerometers, and muscle activity with surface EMG. Information on stimulus pitch motion was available sequentially. Pitch movements of the support surface were best coded in amplitude by ankle rotation velocity, and by head vertical linear acceleration, which started at $13 \mathrm{~ms}$ after the onset of ankle rotation. EMG SL reflex responses in soleus with onsets at $46 \mathrm{~ms}$ provided a distal proprioceptive correlate to the pitch motion. Roll information on the stimulus was available simultaneously. Hip adduction and lumbo-sacral angular velocity were represented neurally as directionally specific short latency stretch and unloading reflexes in the bilateral gluteus medius muscles and paraspinal muscles with onsets at $28 \mathrm{~ms}$. Roll angular accelerations of the head coded roll amplitude and direction at the same time (31 ms). Significant differences in amplitude coding between vestibular loss subjects and controls were only observed as a weaker coding between stimulus motion and head roll and head lateral linear accelerations. The absence of vestibular inputs in vestibular loss subjects led to characteristic larger trunk in motion in roll in the direction of tilt compared to pitch with respect to controls. This was preceded by less uphill flexion and no downhill extension of the legs in vestibular loss subjects. Downhill arm abduction responses were also greater. These results suggest that in man vestibular inputs provide critical information necessary for the appropriate modulation of roll balance-correcting responses in the form of stabilising knee and arm movements. The simultaneous arrival of roll sensory information in controls may indicate that proprioceptive and vestibular signals can only be interpreted correctly when both are present. Thus, roll proprioceptive information may be interpreted inaccurately in vestibular loss subjects, leading to an incorrect perception of body tilt and insufficient uphill knee flexion, especially as cervico-collic signals 
appear less reliable in these subjects as an alternative sensory input.

Keywords Human balance control - Vestibular loss . Proprioception · Muscle activity $\cdot$ Leg flexion responses · Stretch reflex

\section{Introduction}

When human stance is perturbed, muscle responses are generally strong enough to correct for the effects of the perturbation. Upright stance is regained without the need of a stepping reaction. These responses are elicited simultaneously in many body segments with delays of 90-120 ms (Diener et al. 1988; Henry et al. 1998; Allum et al. 1995, 2002). Such "balance correcting responses" are preceded by stretch and unloading reflex responses at the ankle and hip joints with onsets ranging from 25 to $80 \mathrm{~ms}$ (Bloem et al. 2000, 2002; Allum et al. 2003). Even earlier, forces on the sole of the foot (MacPherson 1988; Ting and MacPherson 2004; Perry et al. 2000) and accelerations of the head (Allum and Pfaltz 1985; Runge et al. 1998; Carpenter et al. 1999) provide some information on the amplitude and direction of the perturbation. In order to generate appropriate responses, two processes are required. First the response appropriate for the direction of the perturbation must be triggered, and second the response must be scaled in amplitude according to the perturbation size taking into account that each muscle response has a directional tuning usually aligned along its line of action (MacPherson 1988; Henry et al. 1998; Carpenter et al. 1999). Although considerable research exists on the contributions of proprioceptive and vestibular sensory inputs to the aforementioned response generation processes for the anterior-posterior (AP) direction, (Diener et al. 1988; Allum and Honegger 1998; Bloem et al. 2000; Runge et al. 1998) only timing of proprioceptive inputs but not amplitude relationships have been described for lateral directions (Carpenter et al. 2001; Bloem et al. 2002). The question arises whether both timing or amplitude relationship of sensory inputs are different between roll and pitch especially as the spatio-temporal coupling of balance corrections is different between the roll and pitch directions (Grüneberg et al. 2004, 2005).

Although it is quite well known in both animals and humans how proprioceptive inputs recruit muscle responses (Katz and Pierrot-Deseilligny 1999), very little is known about the corresponding process for vestibular inputs. The two different pathways, the vestibulospinal and reticulospinal tracts, excite motoneurons directly, but more extensively via indirect pathways (Wilson and Yoshida 1969; Grillner and Hongo 1972; Peterson et al. 1980). However, the relationship of how inputs from various semicircular canals are distributed to these pathways is currently unknown (Wilson and Schor 1999). The information available is based on slow and rapid tilts of animals in roll and pitch planes (Pompeino 1984; Anderson et al. 1977), vertical accelerations (Lacour et al. 1979), and translations (Inglis and Macpherson 1995). The information that is available in humans suggests that the central control of responses to roll and pitch perturbations is separately organised (Carpenter et al. 2001; Grüneberg et al. 2005). Most of what is known about the effect of vestibular loss on this response organisation concerns pitch perturbations (Allum and Pfaltz 1985; Allum and Honegger 1998; Runge et al. 1998; Peterka et al. 2004), even though roll instability is greater in vestibular loss subjects (Carpenter et al. 2001). Remarkably translation responses in the pitch plane appear to be little influenced by vestibular loss (Horak et al. 1990; Allum et al. 1993).

In the current paper we investigated the timing and amplitude relation of initial ankle, knee, hip and lumbo-sacral joint motion in pitch and roll planes to both directional and amplitude aspects of the stimulus in order to focus on those signals that most likely to contribute sensory inputs to balance corrections. Our goal was to provide new insights into triggering and modulating aspects of proprioceptive and vestibular signals required for generating balance corrections in the roll and pitch planes. We assumed, as others have done (Diener et al. 1988; Bloem et al. 2000), that a significantly robust correlation between rotation velocity of a joint and short latency (SL) stretch reflex activity in a muscle spanning the joint would imply that central balance control centres (BCC) would receive similar proprioceptive information specifically at times dependent on transmission times of the fastest (Ia) afferents involved. During stimulus presentation, head accelerations were measured with sets of biaxial accelerometers thereby providing information on signals sensed by vestibular sensory systems. One of our two working hypotheses was that differences in the timing of proprioceptive and vestibular sensory signals for the roll and pitch directions, and therefore differences in processing required (parallel versus sequential), is one underlying cause of greater roll than pitch instability in vestibular loss subjects (Carpenter et al. 2001). That is we assumed that in the case of parallel processing both signals must be present for a body-in-space signal to be computed correctly (Mergner et al. 1997). By comparing the onsets and amplitude coding of biomechanical and SL EMG responses of subjects with and without functioning vestibular inputs we hoped to provide supporting evidence for this hypothesis and for the effect of absent vestibular information on roll directed balance corrections. Our second hypothesis was that initial head roll motion is poorly correlated with stimulus motion in vestibular loss subjects preventing the use of cervico-collic signals as a replacement head on trunk signal. 
A more simplified version of this hypothesis would be that the weighting given to proprioceptive inputs from the trunk would not be sufficiently increased to account for the absent vestibular inputs. Thus in the case of vestibular loss, this hypothesis would imply that subjects would respond as if the support surface rolled less.

The insights this approach provided on how roll sensory signals may be combined may explain the distinct roll instability of vestibular loss subjects we observed. We now provide biomechanical data supporting inadequate uphill knee flexion and downhill knee extension in vestibular loss subjects as cause for their roll instability, possibly as a result of the incorrect hypometric perception of body tilt in space. We also show that the trunk and head roll in the opposite direction that of support-surface tilt in humans. In quadripeds the trunk and pelvis rolls in the direction of tilt (MacPherson et al. 2007). Thus the action of vestibulo-spinal influences on ipsi-tilt leg flexion and contra-tilt leg extension would appear to be opposite in polarity in humans to that assumed for quadripeds based on classical animal work (Lindsay et al. 1976; Wilson et al. 1986; Wilson and Melvill Jones 1979; Roberts 1995).

\section{Methods}

\section{Subjects}

We examined 6 subjects ( 5 males and 1 female) with bilaterally absent vestibular function (VL) and 12 control subjects of the same mean age (42 years) and sex ( 2 female). The VL subjects had acquired their sensory loss idiopathically as adults at least 10 years prior to this experiment. VL was characterized by no response (slow phase eye velocity less than $2 \%$ ) to bi-thermal caloric irrigation (100 cc water for $30 \mathrm{~s}$ ) of each ear and by horizontal and vertical vestibulo-ocular reflex responses to whole body rotations of $80^{\circ} / \mathrm{s}$ which were smaller than the lower $1 \%$ bound of normal reference values (Allum and Ledin 1999). These subjects were free from any neurological or previous orthopaedic abnormalities as verified by extensive questioning and a physical evaluation.

All subjects were asked for witnessed, written consent according to the Declaration of Helsinki before participating in the experiments. The Institutional Review Board of the University Hospital in Basel approved the study.

\section{Protocol}

Balance control was examined in a similar way as in previous studies (Carpenter et al. 2004; Allum et al. 2003). Briefly, subjects stood barefoot with their feet lightly strapped into heel guides fixed to the surface of a dual-axis rotating platform. Foot straps were used to ensure that the ankle joints of the subjects remained in the same alignment with the axes of the platform throughout the experiments. Thus the subjects used in-place balance correcting strategy and not stepping reactions to correct for the support-surface tilt. Stance width was the same for all subjects $(14 \mathrm{~cm})$. Subjects were requested to stand with their arms hanging by their sides. Two handrails (adjusted to the height of the wrist of each subject) were located $40 \mathrm{~cm}$ from the sides of the platform centre. Two assistants (one behind and one to the side of the subjects) were present to lend support in case of a fall.

Perturbations to upright stance were delivered by rotating the platform randomly in one of eight different directions with a constant velocity of $60 \%$ and constant amplitude of $7.5^{\circ}$. The perturbation directions were forward (toes-down or $0^{\circ}$ ), backward (toes-up or $180^{\circ}$ ), right $\left(90^{\circ}\right.$ ), left $\left(270^{\circ}\right)$ and four combinations of pitch and roll including forward right $\left(45^{\circ}\right)$, backward right $\left(135^{\circ}\right)$, backward left $\left(225^{\circ}\right)$ and forward left $\left(315^{\circ}\right)$. The first trial was backward (as this direction was the most startling) and excluded from further analysis to reduce habituation effects (Keshner et al. 1987). Then each of the eight perturbation directions was presented randomly eight times under eyes open conditions. Under a subsequent eyes closed series, each of the eight directions was presented randomly five times. Both eyes closed and eyes open tests were employed as not all characteristics of balance corrections recorded to date (see Carpenter et al. 2001) in VL subjects are different from controls when recorded with eyes open. To minimize fatigue, all participants were given a 2-3 min seated rest after the 20th, 40th, and 65th trial of the series. Each perturbation was preceded by a random 5-20 s delay. During this delay, visual feedback of the subject's own low-pass filtered anterior-posterior (AP) and medio-lateral (ML) ankle torques was presented to the subject on a cross with light emitting diodes at $4 \mathrm{~m}$ from the subject. This visual feedback was used by the subjects to standardise prestimulus position across trials for each individual, being pre-set to values obtained at a comfortable stance prior to each series. This feedback was switched off for $5 \mathrm{~s}$ at stimulus onset. For eyes closed trials, auditory feedback was provided as well.

\section{Data collection}

Recordings of all biomechanical and electromyographical (EMG) data commenced $100 \mathrm{~ms}$ prior to perturbation onset and were collected for $1 \mathrm{~s}$.

Full body kinematic data was collected using a 3D optical tracking system with 21 infrared emitting diodes (IREDs) (Optotrak, Northern Digital). The Optotrak cameras sampled the signals from the IREDs at $64 \mathrm{~Hz}$ and were 
placed approximately $4 \mathrm{~m}$ in front of the subjects. The infrared emitting diodes were placed bilaterally on the following anatomical landmarks: frontally at the level of the lateral malleolus (ankle); at the centre of the patella (knee); frontally at the level of the greater trochanter (hip); at the anterior superior iliac spine (pelvis); elbow axis; acromion (shoulder); processus styloideus (wrist); and on a tight head band (see accelerations below) placed just above the level of the ears. In addition, a diode was placed on the chin (also head) and one on the angulus sterni (upper trunk). Three IREDs were placed at the front corners and on the left side centre of the platform to define pitch and roll movements of the platform, which were also measured directly with potentiometers sampled at $1.024 \mathrm{kHz}$. All subjects wore tight fitting shorts and vests to prevent marker movements.

Head linear and angular accelerations were computed using analog signal processing from the outputs of four dual axis linear accelerometers, (Entran) with ranges of $\pm 5 \mathrm{~g}$, each mounted at $90^{\circ}$ separation on a lightweight, adjustable, tight head-band. Head vertical linear acceleration was computed, for example, from the sum of all four accelerometer signals with a vertical component, whereas head roll acceleration was computed from the difference in vertical linear accelerations at the ears. Accelerometers were adjusted to be in the gravity plane at the start of the experiment, but not corrected thereafter. All analog computed signals were sampled at $1 \mathrm{kHz}$. Only one acceleration, lateral linear acceleration was computed based on three head markers using our motion analysis system.

EMG recordings were obtained using previously described techniques. (Carpenter et al. 1999; Grüneberg et al. 2005). In order to record EMG signals, pairs of silversilver chloride electrodes were placed approximately three $\mathrm{cm}$ apart along the muscle bellies of; left tibialis anterior, left soleus, left and right gluteus medius, left and right paraspinals at the L1-L2 level of the spine, left biceps and left medial deltoid muscles. EMG amplifier gains were kept constant and pairs of electrodes and lead lengths assigned to individual muscles were not changed between subjects. EMG signals were recorded using a pre-amplifier with a band-pass $0.7 \mathrm{~Hz}$ to $2.5 \mathrm{kHz}$, then amplified before stages of band-pass analog filtering between 60 and $600 \mathrm{~Hz}$, full wave rectification, and low pass filtering at $100 \mathrm{~Hz}$ with a 3rd-order Paynter filter prior to sampling at $1 \mathrm{kHz}$.

Data analysis

Following analog to digital conversion of the data, all biomechanical and EMG signals were averaged offline across each perturbation direction. Subject averages were pooled to produce population averages for a single direction. Zero latency was defined as the onset of platform rotation measured with the potentiometer system. By comparing the
Optotrak and potentiometer recordings of support-surface displacement the appropriate time shift could be applied to the Optotrak data to align it with EMG and head acceleration records (see Fig. 1).

\section{Kinematic analysis}

Position data from the Optotrak recordings was digitally filtered at $16 \mathrm{~Hz}$ using a zero phase shift fourth order Butterworth low-pass filter. Knee and elbow angles were calculated as the angle spanned by the upper and lower segments of the legs and arms, respectively. Absolute rotation angles of the planes defined by trunk and pelvis body segments and the platform surface were defined using 3 or 4 markers on these segments. A rotation matrix was used to calculate the rotation of these segments in the pitch (sagittal) plane and roll (frontal) plane. Rotations of the upper arm with respect to the trunk and the upper leg with respect to pelvis were calculated as ball joint angles defined by two angles, for example, for the leg by hip flexion and abduction. Stimulus induced changes were calculated with respect to averaged angles over a pre-trigger time interval of $90 \mathrm{~ms}$ ending $10 \mathrm{~ms}$ prior to the stimulus onset. For each segment, angle changes were calculated at both 300 and $800 \mathrm{~ms}$. The measurement at $300 \mathrm{~ms}$ was chosen to quantify the impact of the balance correcting responses on the kinematics. At $800 \mathrm{~ms}$ controls had reached more or less their stable end position. Angular velocity at each joint was calculated after differentiation of the angles with respect to the time and then resampling the data at $512 \mathrm{~Hz}$ after fitting four true samples with a cubic spline. Head lateral linear acceleration was obtained on repeating the differentiation process on head position data. Although the sampling rate was lower for the Optotrak compared to analog recorded data from accelerometers and potentiometers the accuracy measured using the SD of the measurements was higher (see Fig. 1). Differences in joint velocities of VL and control subjects were investigated at time points when these signals diverged most between the two sample populations.

In order to calculate Pearson correlations between joint angular velocity and stimulus amplitudes we systematically measured joint velocity over a $10 \mathrm{~ms}$ window that commenced $40 \mathrm{~ms}$ from the time point when velocity traces across directions first diverged from one another for more than $20 \mathrm{~ms}$. The time of trace separation generally increased for links located further from stimulus i.e. proximal joints. For velocity traces these times set at $5 \mathrm{~ms}$ after stimulus onset for the ankle joint, $23 \mathrm{~ms}$ for the knee, $28 \mathrm{~ms}$ for the hip flexion, $20 \mathrm{~ms}$ for hip adduction, and $40 \mathrm{~ms}$ for arm adduction. For head acceleration traces the separation times were set at $10 \mathrm{~ms}$ for head vertical acceleration, $26 \mathrm{~ms}$ for head pitch acceleration and $40 \mathrm{~ms}$ for head roll acceleration. For analog sampled head accelerations (and 
Responses for back right $\left(135^{\circ}\right)$
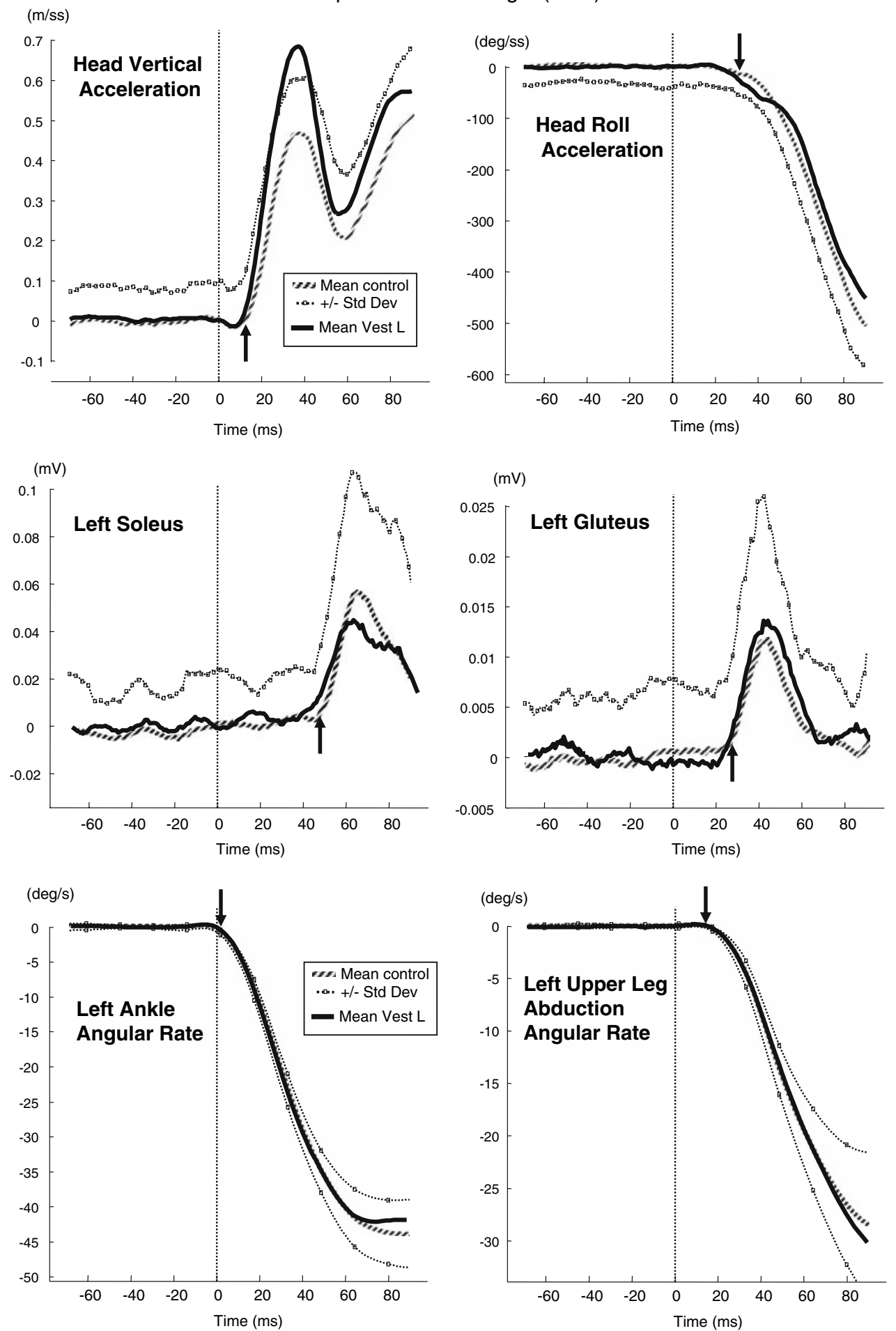

Fig. 1 Population responses of control and bilateral vestibular loss $(V L)$ subjects under eyes open conditions for a combined pitch and roll directed $\left(135^{\circ}\right.$ direction, backward right) tilt of the support surface. Each of the sets of responses shows the mean and standard deviation for 12 control subjects and the mean of 6 VL subjects. Each subject response was repeated 8 times. Data $60 \mathrm{~ms}$ prior to and $80 \mathrm{~ms}$ after stimulus onset is shown. The vertical dotted line at $0 \mathrm{~ms}$ marks the onset of

the stimulus (first deflection of platform velocity). The sample times are shown on the mean plus standard deviation of the control traces by open circles. The upper 4-set of traces were sampled directly as analog signals at 1,024 Hz. The ankle angle flexion velocity and hip adduction velocity were based on samples at $64 \mathrm{~Hz}$ from the digitally based motion analysis system. The mean onsets (determined visually) of each trace from pre-stimulus values are marked on the traces 
digitally computed head lateral linear acceleration) we used the same $10 \mathrm{~ms}$ window but set it $20 \mathrm{~ms}$ not $40 \mathrm{~ms}$ after the divergence of traces. The windows used for these purpose are marked in Figs. 2, 3 and 5.

\section{EMG analysis}

EMG responses were first corrected for background activity by subtracting from the responses the average level of activity measured over a $90 \mathrm{~ms}$ period ending $10 \mathrm{~ms}$ prior to perturbation onset. Then EMG areas (integrals) were calculated using trapezoid integration.

For the purposes of calculating EMG areas (integrals) of balance correcting responses, the procedure described in Grüneberg et al. (2005) was employed. First, onset latencies of these responses were calculated for the perturbation direction that generated the largest balance correcting EMG response. This direction was determined for each muscle separately by identifying the maximum peak amplitude for the population response of the muscle
Fig. 2 Pitch-directed leg responses of control subjects. Average platform ankle, knee and hip flexion pitch angular velocity and soleus EMG (short latency) SL stretch reflex responses of controls under eyes open conditions are shown. Each of the traces is an average for the 12 subjects, each stimulus was repeated 8 times to yield a total of 96 responses. The first $140 \mathrm{~ms}$ of the responses are shown for 5 directions $\left(0^{\circ}, 45^{\circ}, 90^{\circ}, 135^{\circ}\right.$, $\left.180^{\circ}\right)$ of support-surface tilt with different pitch amplitude ( 6 deviations for soleus EMG) as indicated by the inserted legend. The vertical dotted line marks the onset of the stimulus tilt (first deflection of platform velocity). The pitch and roll stimulus profiles are shown in the lower sets of traces in Fig. 3. The biomechanical data was recorded with the motion analysis system and then differentiated to yield velocity. The intervals used for the regression analysis of the plotted variables in the figure with pitch stimulus amplitude are marked by rectangular boxes. The response for the direction 225 has been added to the soleus response in order to show its response asymmetry

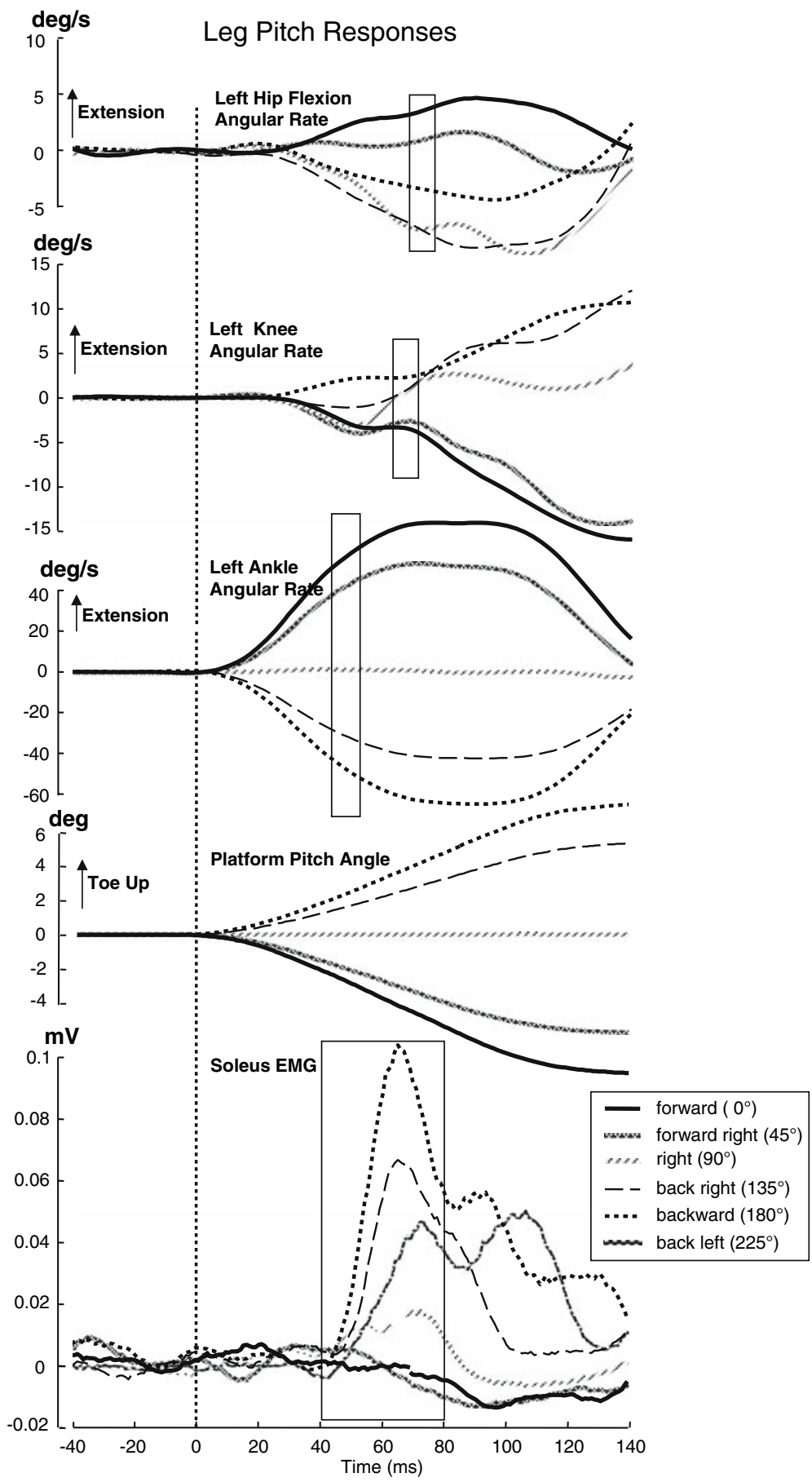




\section{Head Acceleration Responses}
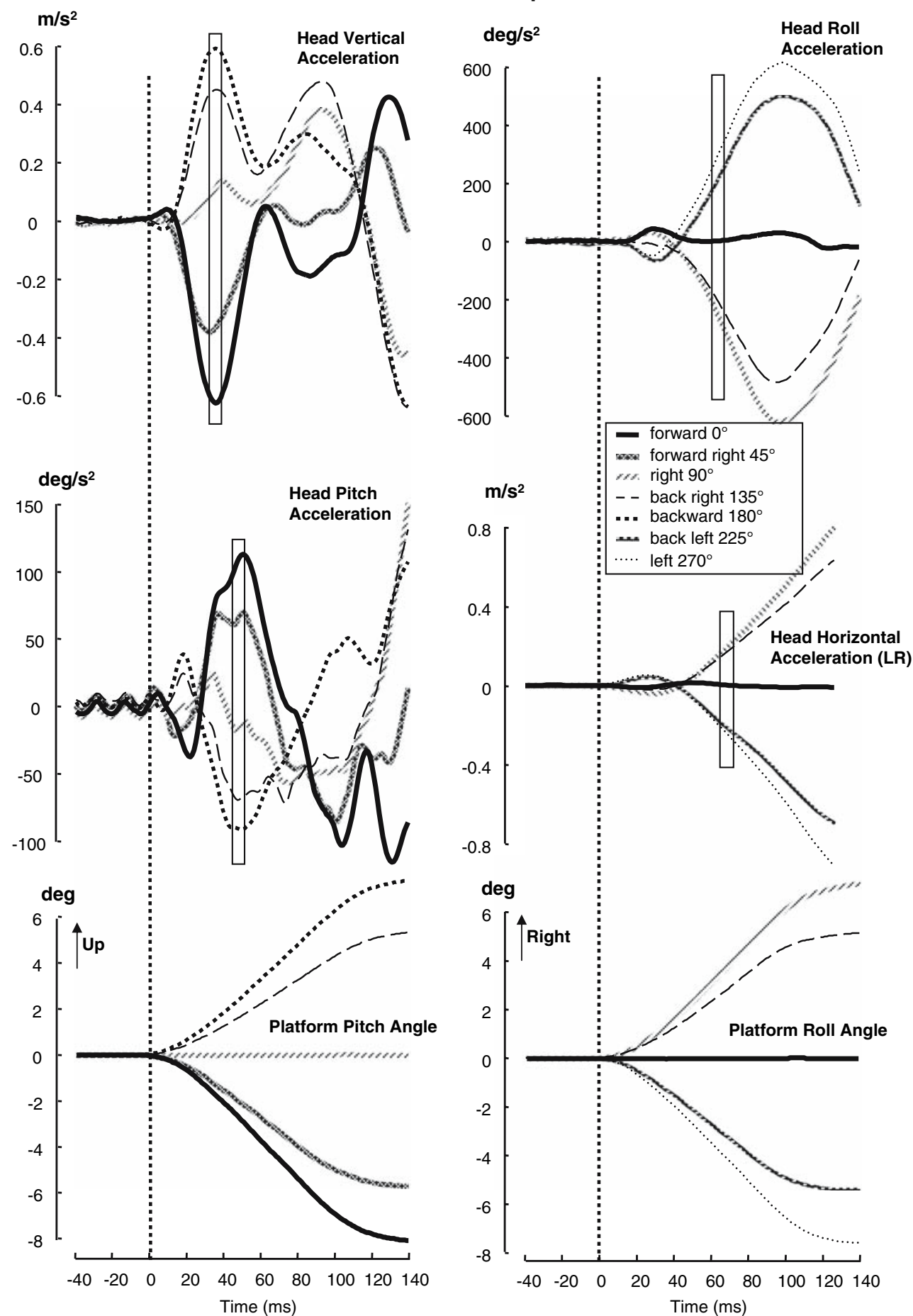

Fig. 3 Population head acceleration responses of control subjects (eyes open condition). The head vertical linear and pitch angular and head roll acceleration were recorded with accelerometers at a sampling rate of $1 \mathrm{kHz}$. Lateral linear acceleration was computed from a double differentiation of head marker position recorded with the motion analysis system at $64 \mathrm{~Hz}$. The intervals used for the regressions of Fig. 4

within pre-defined intervals (leg muscles: 100-250 ms; gluteus $60-300 \mathrm{~ms}$; paraspinals: $100-200 \mathrm{~ms}$; arm muscles: $20-240 \mathrm{~ms}$ ). For the direction with the maximal

are shown by rectangular boxes. Note the early peak in head vertical acceleration and more gradual increase in head roll and lateral linear accelerations. The stimulus profiles of the support surface movement recorded with potentiometers are shown in the bottom two sets of traces. The deviations of support surface tilt are indicated by different line types as indicated in the inserted legend

response, onset latencies were defined as the first sample prior to when the EMG activity was less $10 \%$ of the peak amplitude after correction for mean background muscle 
activity. This onset was set as the beginning of the integration interval across all directions for that muscle. The end of the integration interval for each muscle was determined by examining when activity after the peak response first decreased to $15 \%$ of the peak amplitude. This procedure yielded in controls an integration interval of $87 \mathrm{~ms}$ commencing at $171 \mathrm{~ms}$ on average for soleus, an interval of $119 \mathrm{~ms}$ commencing on average at $122 \mathrm{~ms}$ for paraspinals and an interval of $51 \mathrm{~ms}$ commencing at $122 \mathrm{~ms}$ for deltoid. These average onset times and integration intervals in controls were then used as fixed intervals to compare EMG responses between controls and VL subjects. Fixed integration intervals were used for short latency (SL) stretch (and unloading) reflexes without prior determination of average onset times as these responses were considerably smaller. For the SL stretch reflexes the intervals were; $40-80 \mathrm{~ms}$ for soleus, and $25-65 \mathrm{~ms}$ for gluteus and paraspinals (as shown in Figs. 2, 5).

\section{Statistics}

Our primary analyses concentrated on between-groups comparison of VL patients and controls using a repeated measures ANOVA model (group $\times$ direction $\times$ vision) for both EMG and kinematic data. The 8 directions were reduced to 5 by pooling data for the directions of 45 and 315,90 and 270, and 135 and 225 together for biomechanical data based on the well-tested assumption of left-right asymmetry in control data (Carpenter et al. 1999, 2001, 2004). Kinematic amplitudes for roll responses to the left were inverted prior to pooling. EMG response areas were analysed statistically using the three perturbation directions of greatest activity for each muscle (forward and forward roll directions for soleus, backward and backward roll directions for paraspinals, gluteus medius and deltoid). Significant main and interaction effects were further explored using post hoc Bonferroni $t$ test. Significance levels were set at 0.05 .

\section{Results}

Proprioceptive and vestibular information coding support-surface pitch and roll tilt

A combined pitch and roll tilt, such as that shown in Fig. 1, revealed response characteristics, some of which were more related to the pitch component, others more to the roll component of the stimulus. We therefore analysed early timing and amplitude relationships of biomechanical and EMG responses separately for roll and pitch in controls and vestibular loss subjects. This analysis in roll and pitch directions was performed to gain insights into possible directionally based sensory integration deficits culminating in balance instability of vestibular loss (VL) subjects.

Correlations to pitch components in initial joint movements

Backward pitch (and combined backward pitch and roll) perturbations initiated a dorsi-flexion of the ankle joint, followed by extension of the knees and flexion at the hip (Figs. 1, 2), and then lumbo-sacral joint motion. Forward pitch perturbations also initiated a distal to proximal pattern of joint movement, however the ankles were initially plantar-flexed, followed by knee flexion, a small amount of hip extension (Fig. 2) and then a negligible lumbo-sacral extension. As Fig. 2 indicates ankle pitch angular velocity was highly correlated $(r=0.99)$ with platform pitch rotation in controls. The correlation was equally high for VL subjects $(r=0.99)$. Knee flexion velocity provided a more variable response with platform pitch rotation $[r=0.39$ in controls, $r=0.2$ in VL subjects (not significant)], as did flexion at the hip (upper leg with respect to the pelvis, $r=0.39$ in controls, $r=0.58$ in VL subjects). Lumbo-sacral joint correlations with pitch were not significant for either group.

Head accelerations were also specific to perturbation direction, with initial head accelerations observed in forward rotation and upward direction for backward perturbations, and backward rotation and downward direction for forward perturbations (Figs. 1, 3 left traces). Vertical linear acceleration of the head had an onset at $13 \mathrm{~ms}$ (value for $135^{\circ}$ direction, $12.9 \pm 4.5 \mathrm{~ms}$, see Fig. 1 ) in both controls and VL-subjects and had a peak between 30 and $40 \mathrm{~ms}$ that was well correlated with the pitch stimulus amplitude (see Figs. 1, 3 and 4) in controls $(r=0.9)$ and VL subjects $(r=0.96)$. A trend for larger vertical head linear accelerations was noted in VL subjects (Figs. 1, 4). Pitch angular accelerations of the head had an onset latency at ca. $25 \mathrm{~ms}$, peaked at $50 \mathrm{~ms}$ and had an amplitude that was less well correlated with pitch stimulus amplitude ( $r=0.60$ in controls, 0.76 in VL subjects) than vertical linear acceleration (Figs. 3, 4). Prior to $100 \mathrm{ms,} \mathrm{yaw} \mathrm{angular} \mathrm{accelerations} \mathrm{of}$ the head were negligible for the combined roll and pitch stimuli, as were anterior-posterior linear accelerations.

\section{Correlation to roll components in initial joint movements}

Initially the roll stimulus created by support surface lateral tilt, drove the uphill leg upwards, causing a roll of the pelvis in the same direction of the platform movement, while the trunk rotated in the opposite direction (to both the pelvis and platform movements) due to joint coupling (Figs. 5, 8).

The greatest correlations for roll perturbations were observed in the proximal joints (Figs. 5, 6). The roll rotation of the hip joint, commencing just prior to $20 \mathrm{~ms}$ (value for $135^{\circ}, 17 \pm 3 \mathrm{~ms}$, see Fig. 1) in controls and VL 


\section{Regressions Head Accelerations}

Head Vertical Linear Acceleration $\left(\mathrm{m} / \mathrm{s}^{2}\right) 30-40 \mathrm{~ms}$

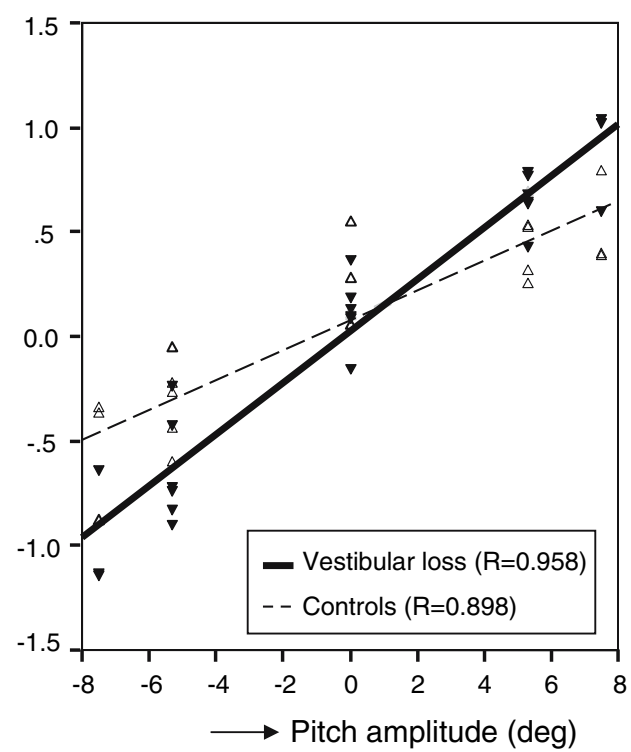

Head Pitch Angular Acceleration (deg/s²) 45-55 ms

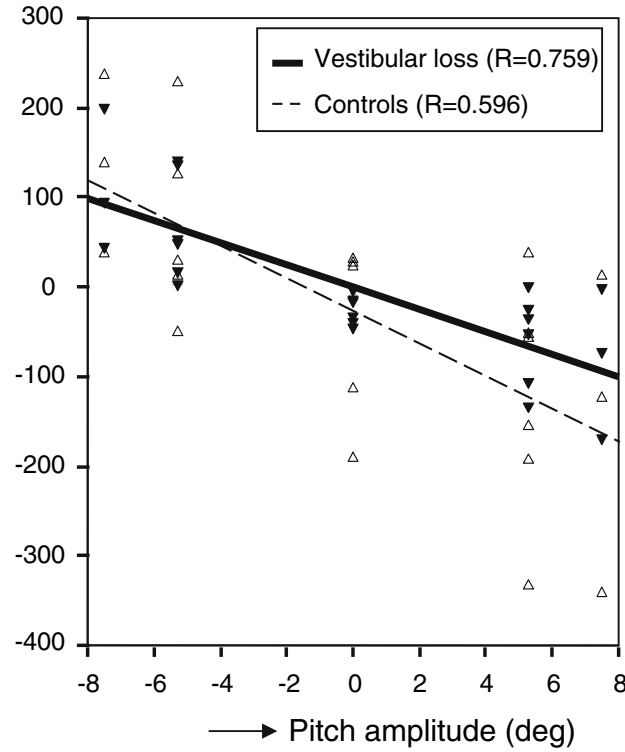

Fig. 4 Regressions between head angular or linear accelerations and stimulus amplitudes for control and VL subjects. The measurement intervals were $10 \mathrm{~ms}$ in duration starting $20 \mathrm{~ms}$ after acceleration onset and are indicated in each panel. The measurements for each subject's eyes closed and eyes open responses have been pooled as no effect of vision was noted for these variables. Data from 12 normal subjects and

subjects, followed by the lumbo-sacral joint were both clearly related to the roll stimulus (Fig. 6) with very high correlation co-efficients observed between support-surface roll angle and roll angular velocity in the hip $(r=0.98$ in controls and 0.93 in VL subjects) and lumbo-sacral joint $(r=0.98$ in controls and 0.91 in VL subjects). Differences in knee velocities yielded a weak, non-significant correla-
Head Lateral Linear Acceleration $\left(\mathrm{m} / \mathrm{s}^{2}\right) 63-73 \mathrm{~ms}$

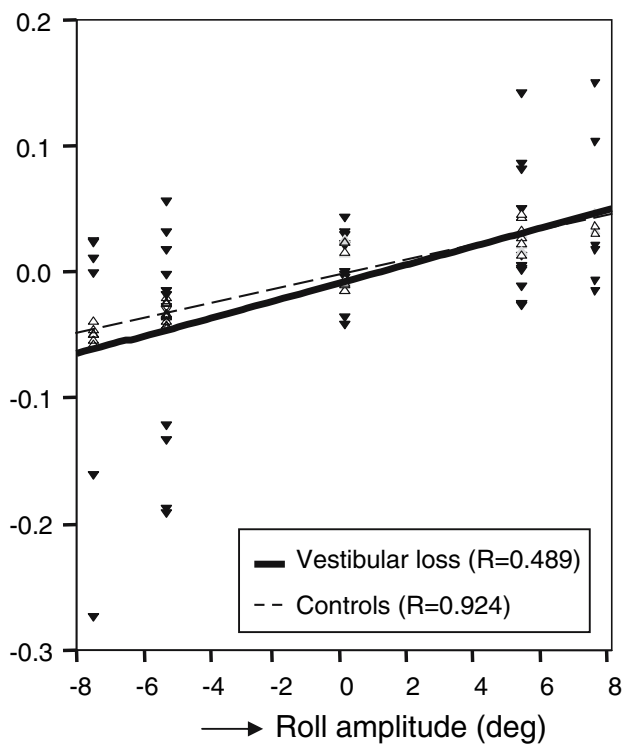

Head Roll Angular Acceleration (deg/s $\left.\mathrm{s}^{2}\right) 60-70 \mathrm{~ms}$

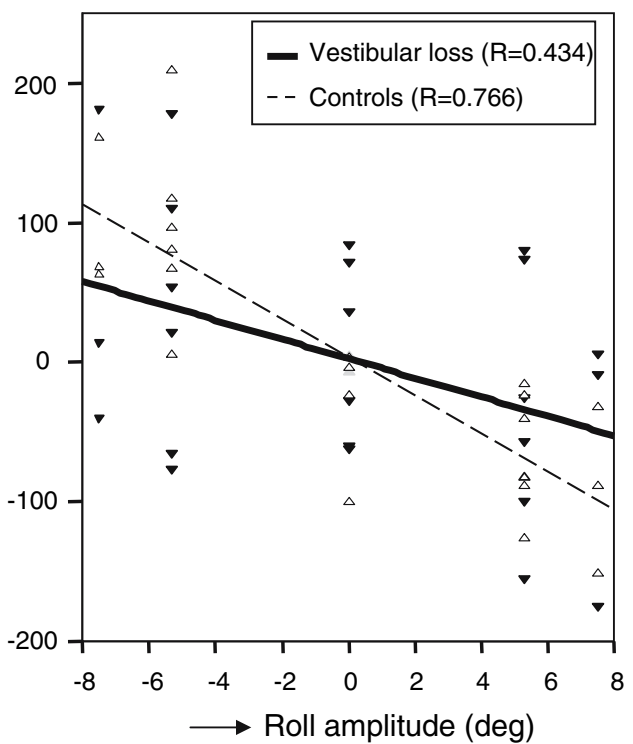

6 VL subjects are shown. Positive pitch is toe-up, negative toe-down. Each left and right forward pitch amplitude yielded two pooled measurement values per subject as did backward left and right pitch. Likewise forward and backward pitch yielded two pooled roll values. Correlation coefficients of the regressions for normal and VL subjects are provided

tion $(r=0.09)$ in both groups. Arm abduction velocities with onsets at $40 \mathrm{~ms}$ were related $(r=0.98$ in controls, 0.94 in VL subjects) to the roll stimulus (see Fig. 5).

In contrast to the early onset of the pitch-related head accelerations, both roll angular acceleration and lateral linear acceleration, as shown in Fig. 1, had later onsets after $25-30 \mathrm{~ms}$ (roll acceleration onset for $135^{\circ}, 31 \pm 9 \mathrm{~ms}$ in 
Fig. 5 Roll-directed upper body responses of controls. Average hip abduction, lumbo-sacral roll abduction and arm biomechanical and muscle EMG responses of controls tested under eyes open conditions are shown. The first $160 \mathrm{~ms}$ of the responses for the 6 directions of support-surface roll tilt $(45,90,135,225$, 270 and 315) with different roll amplitudes are shown. The pelvis, trunk and arm movements were recorded with the motion analysis system. For other details refer to the legends of Figs. 1 and 2. The measurement intervals used for the regressions of Fig. 6 are marked by rectangular boxes on the sets of traces

\section{Upper Body Responses}

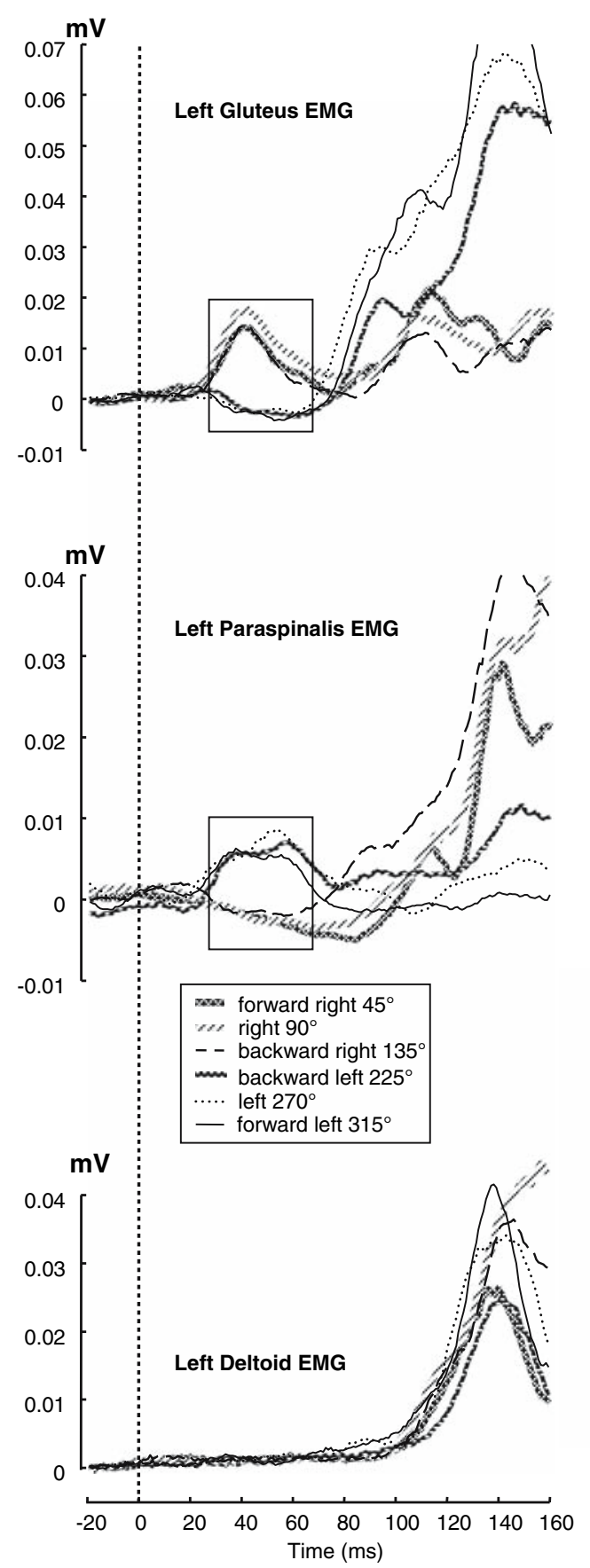

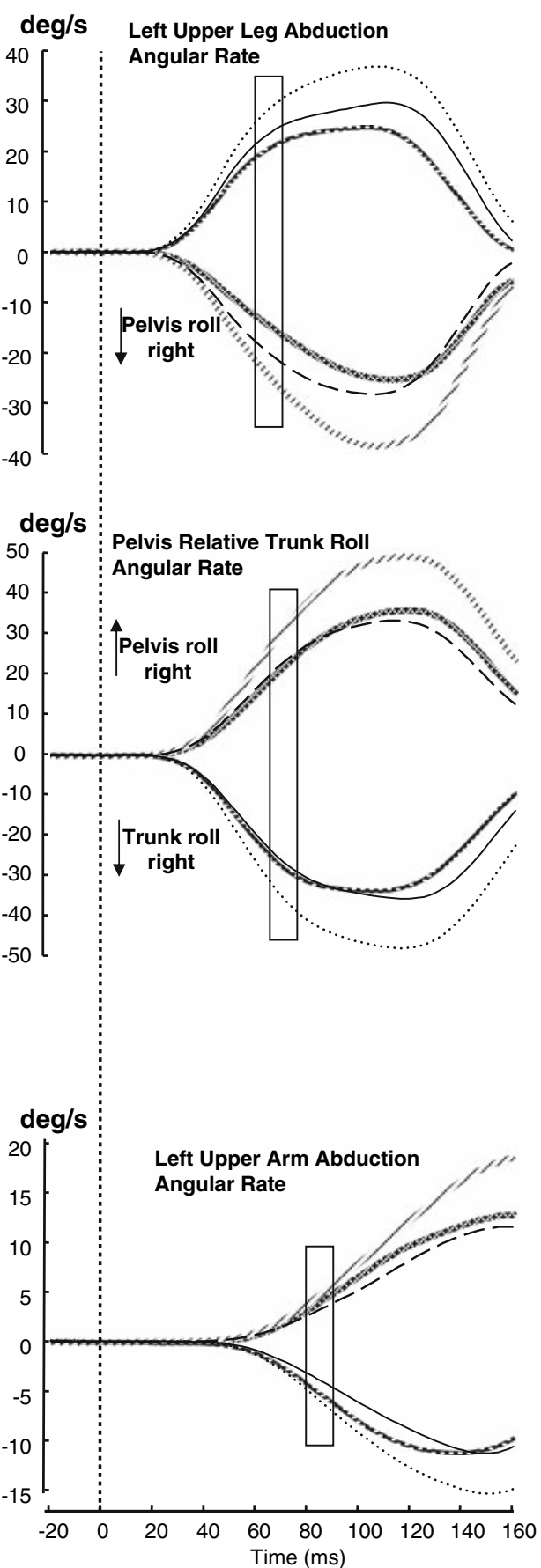

controls) and then began to be clearly stimulus related to the roll amplitude at ca. $60 \mathrm{~ms}$ (Fig. 3). Correlations between head accelerations and roll stimulus amplitude yielded $r$ values of 0.77 for angular roll acceleration and 0.92 for lateral linear acceleration (Fig. 4) in controls, with lower correlations $r=0.43$, respectively $r=0.48$ in $\mathrm{VL}$ subjects. Furthermore there was a trend for lower amplitude of roll acceleration in VL subjects compared to controls for head roll acceleration (Fig. 4).
Short latency stretch reflex responses

Dorsiflexion of the ankle joint produced a short latency (SL) stretch reflex response in soleus that commenced some $45 \mathrm{~ms}$ ( $46 \mathrm{~ms} \pm 6.5 \mathrm{~ms}$ for $135^{\circ}$ see Fig. 1) after onset of the stimulus (Figs. 1, 2) and was not different between controls and VL subjects (Fig. 1). Plantar flexion rotation caused a small unloading response in the same muscle. Left and right backwards rotations $\left(225\right.$ and $\left.135^{\circ}\right)$ did not produce equal stretch 
Fig. 6 Regressions of the left hip and lumbo-sacral joint roll rotation velocities and EMG SL stretch and unloading responses in left gluteus medius and paraspinals muscles against stimulus roll amplitude. Positive roll is to the right. The muscle regression used the response area between 25 and $65 \mathrm{~ms}$ after stimulus onset. This interval is depicted in Fig. 5. Data from the gluteus medius muscle of one control (marked asterisk) was excluded from the regression as it was an outlier. For other details refer to the legend of Fig. 4

\section{Regressions Upper Body Roll Signals}

Left Hip abduction velocity (deg/s) 60-70 ms
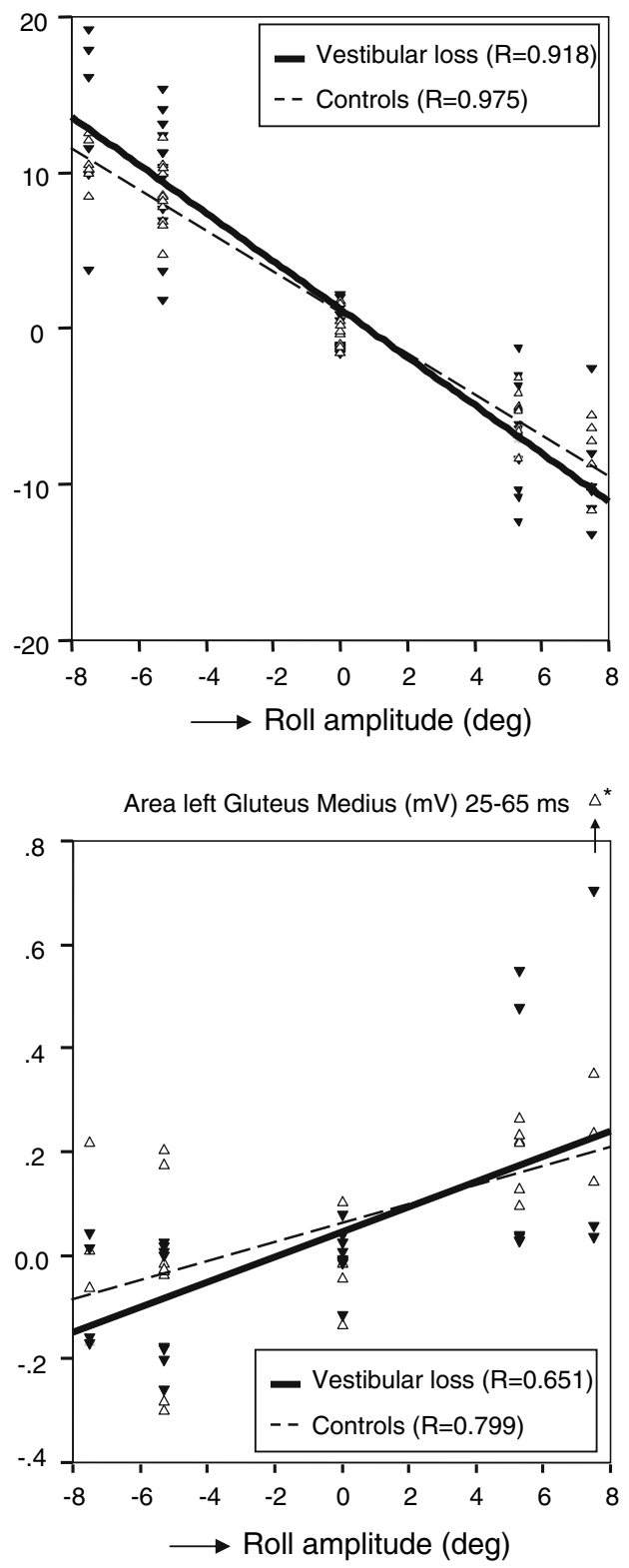

Pelvis relative to Trunk velocity (deg/s) $65-75 \mathrm{~ms}$

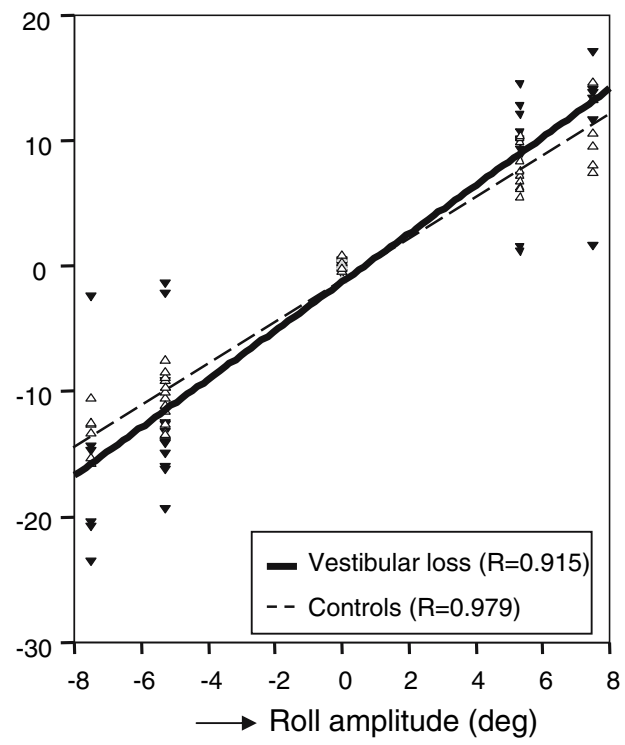

Area Left Paraspinal (mV) 25-65 ms

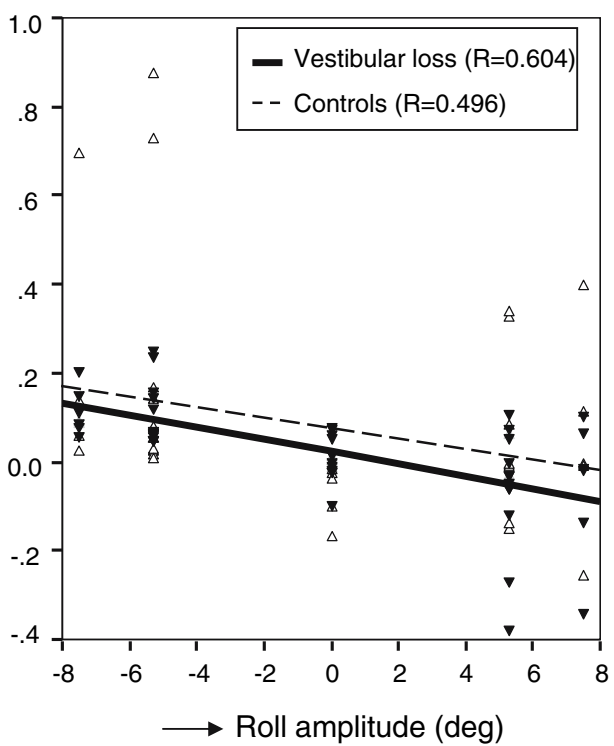

response amplitudes over the first $40 \mathrm{~ms}$ of the response even though the pitch stimulus was identical (Fig. 2). This is presumably due to the anatomical orientation of the soleus muscle's line of maximum stretch responsiveness being aligned medially with respect to a pure pitch rotation. Assuming the CNS would average out this effect for the left and right soleus muscles, we calculated a regression of the area under first $40 \mathrm{~ms}$ of the response against all pitch amplitudes. The regression indicated high correlations between soleus stretch reflexes responses and platform pitch amplitude in both controls $(r=0.81)$ and VL subjects $(r=0.87)$ —see also Fig. 2. The correlation with platform roll was weak in both control and VL subjects $(r<0.2)$.
When the support-surface tipped right, the pelvis also rotated right, causing a relative adduction of the left leg (as shown in Fig. 5), which stretched the left gluteus medius. The trunk rotated to the left, thus shortening the left paraspinals (Fig. 5). The ensuing SL reflexes in gluteus medius (for $135^{\circ}, 27.5 \pm 4.5 \mathrm{~ms}$ in controls, see Fig. 1) and paraspinal muscles had onset latencies that preceded earliest responses in the soleus muscles (compare responses in Figs. 1, 2 and 4). Therefore, even though hip adduction was delayed with respect to the ankle joint flexion (compare joint angular velocity responses in Figs. 1, 2 and 4), roll proprioceptive directional and amplitude information was available for transmission to the CNS, from afferents transmitting early SL 
stretch and unloading responses elicited in gluteus medius and paraspinal muscles (Fig. 5) prior to the information from the ankle joint. Correlations of regressions of the first $40 \mathrm{~ms}$ of the early reflex responses in gluteus medius with roll was 0.8 in controls and 0.94 in VL subjects and thus similar in value to those of soleus for pitch (0.81). SL responses in paraspinals had a lower regression value with roll $(r=0.50$ in controls, $r=0.60$ in VL subjects).

\section{Differences in trunk displacements post $150 \mathrm{~ms}$}

Our observations on trunk motion between controls and VL subjects confirmed those of Carpenter et al. (2001). For example, the stick figures of Fig. 7 confirm the instability of the VL subjects even though initial positions were not different between VL subjects and controls. Findings on trunk motion here are described briefly in order to provide background information supporting our new findings concerning the effect of vestibular loss on leg and arm movements. There was a group by direction interaction for peak trunk angular pitch velocity $\left(F_{(4,60)}=3.5, P=0.01\right)$ and trunk pitch angle at $300 \mathrm{~ms}\left(F_{(4,60)}=2.787, P<0.05\right)$. Thus significantly larger trunk flexion observed in BVL subjects compared to controls for backward pitch $\left(180^{\circ}\right)$ rotation $(P<0.05)$ with no effect of vision. Therefore, eyes open and closed responses have been pooled in Fig. 8. After $400 \mathrm{~ms}$, BVL subjects were observed to have a larger and more rapid extension of the trunk, compared to controls who maintained forward trunk flexion for backwards pitch (Fig. 8).
Trunk roll motion was not influenced by vestibular loss prior to $400 \mathrm{~ms}$ (see Figs. 7, 8). Thereafter, the trunk deviated to the side of platform tilt. Trunk roll angular displacements at $800 \mathrm{~ms}$ were significantly influenced by vestibular loss. At $800 \mathrm{~ms}$, a significant group by direction interaction was observed for trunk roll angle $\left(F_{(4,60)}=6.526\right.$, $P=0.001)$, but no interaction for vision. As shown in Fig. 8, BVL subjects had significantly larger and oppositely directed trunk roll angular displacements compared to controls for all three roll directed perturbation directions $(P<0.05)$.

\section{Leg displacements post $150 \mathrm{~ms}$}

The uphill knee flexion and downhill knee extension movements were different in VL subjects and controls. The stick figures of the leg movements provided in Fig. 9 illustrate the differences for typical subjects from each population. The left, uphill knee of the VL subject is flexed less than the control subject. Furthermore the right, downhill knee is flexed rather than extended in the VL subject compared to the control subject. Our analysis confirmed these observations and revealed differences in both peak knee flexion velocities at approximately $250 \mathrm{~ms}$ and knee angles at $800 \mathrm{~ms}$.

Roll tilt of the support surface resulted in a peak flexion velocity of the uphill knee at ca. $270 \mathrm{~ms}$ post-perturbation onset. Knee peak velocity was found to be significantly influenced by a group (VL vs. control) by direction interaction $\left(F_{(4,60)}=4.2, P<0.001\right)$. As shown in Fig. 10, peak

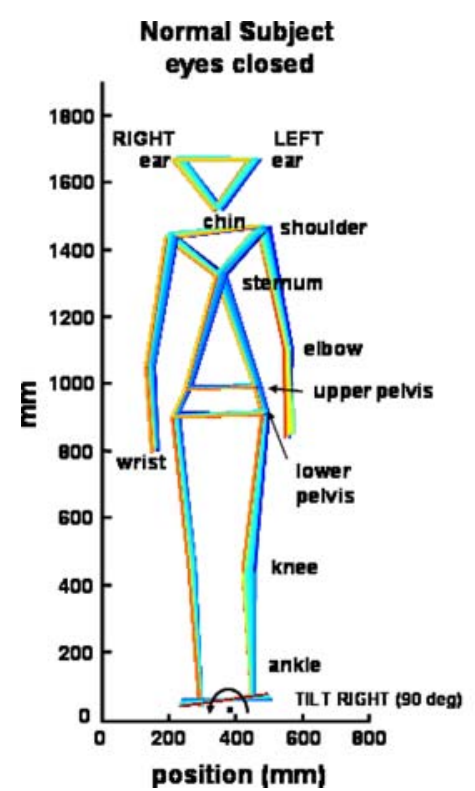

Fig. 7 Stick figure representatives of body segment movements to right tilt of the support-surface in a typical VL and control subject under eyes closed conditions. The stick figures were drawn based on the 18 infrared Optotrak markers on the body. The 64 timeframes, recorded over $1 \mathrm{~s}$, are indicated by the colour code, with blue colours mark-

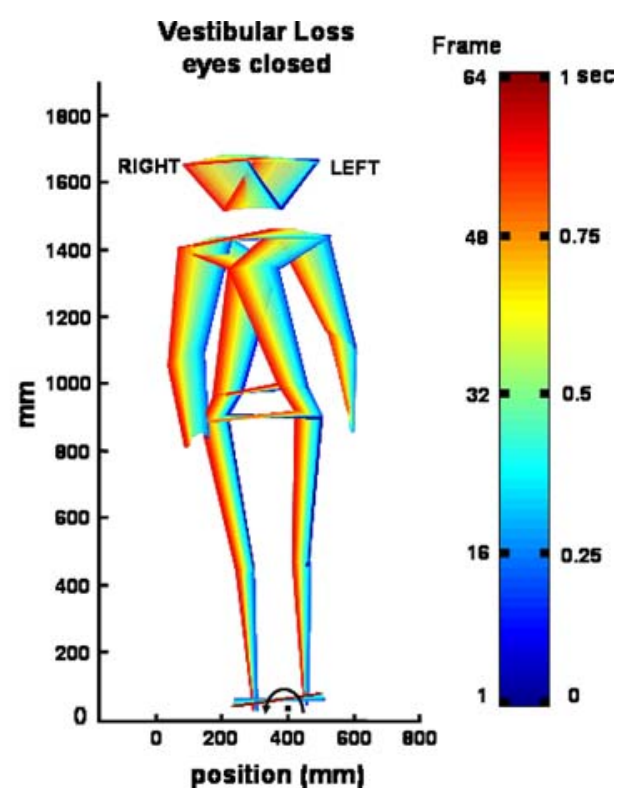

ing the early frames and red colours the late frames. The supportsurface rotation started at frame 6 . The marker positions were averaged for 8 identical stimuli. The views are from in front of the subjects. Note the similar arm and leg positions for the two subjects prior to tilt and tendency of the VL subject to fall right 
Trunk Pitch eyes closed

Backward $\left(180^{\circ}\right)$
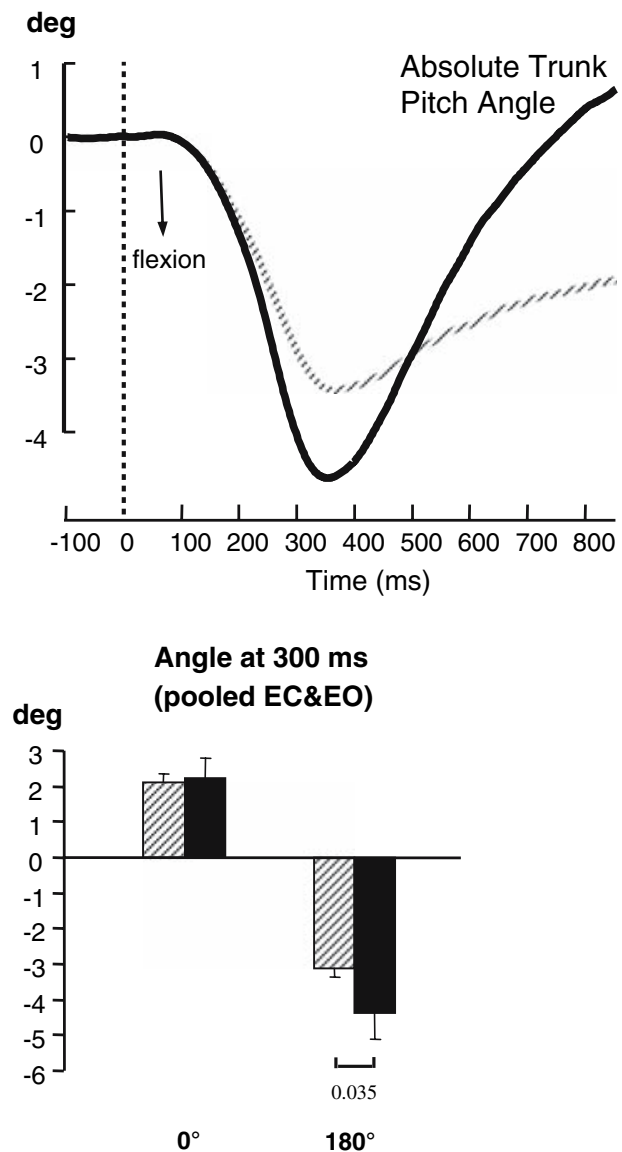

Fig. 8 Trunk pitch and roll responses of control and VL subjects. The mean population traces of trunk pitch and roll are shown for a pure pitch, backwards $\left(180^{\circ}\right)$ and a roll (to the left, $\left.270^{\circ}\right)$ tilt of the supportsurface under eyes closed conditions are shown. The column plots show pooled eyes closed and eyes open means and standard error of
Trunk roll eyes closed

Left $\left(270^{\circ}\right)$
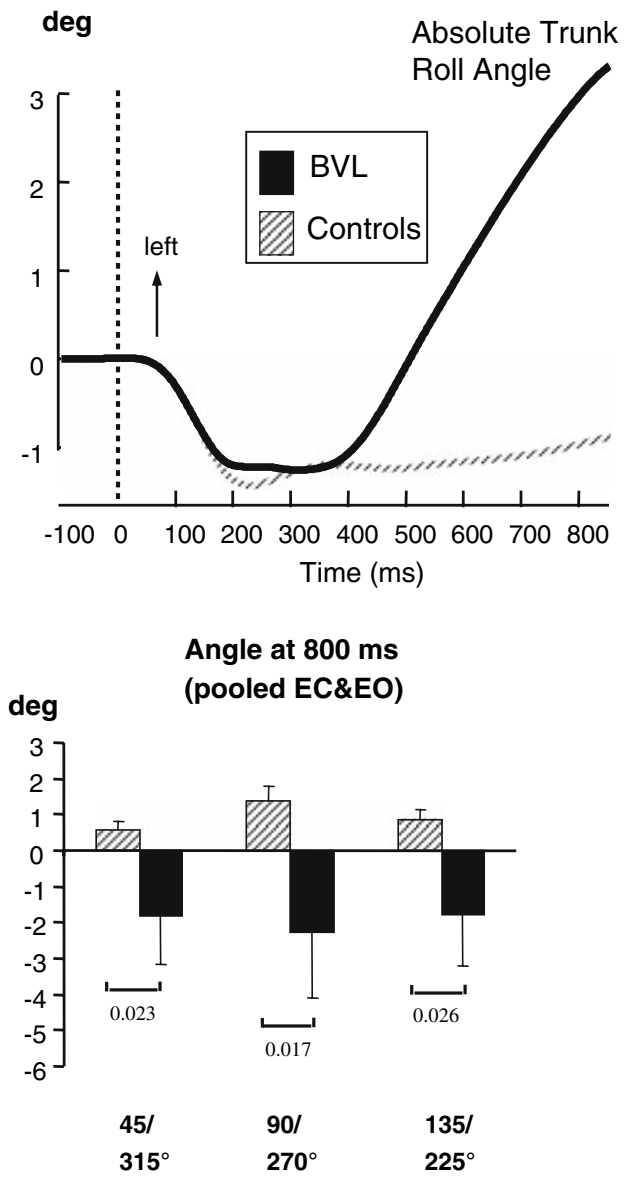

mean (SEM) of trunk pitch angle at $300 \mathrm{~ms}$ and trunk roll angle at $800 \mathrm{~ms}$ after stimulus onset for the VL subjects and controls (no effect of vision was noted therefore the data was pooled). Roll data with equivalent pitch amplitudes has been pooled. Significant differences are marked by a horizontal bar and the $P$ value
Fig. 9 Stick figure representations of knee movements to a right tilt of the support-surface in a typical VL and control subject. Details of the figure are provided in the legend to Fig. 7. The views in the figures are from slightly in front $\left(8^{\circ}\right)$, slightly raised $\left(4^{\circ}\right)$ and from the right. Note the difference in angles of left knee flexion and right knee flexion at the end of the recordings
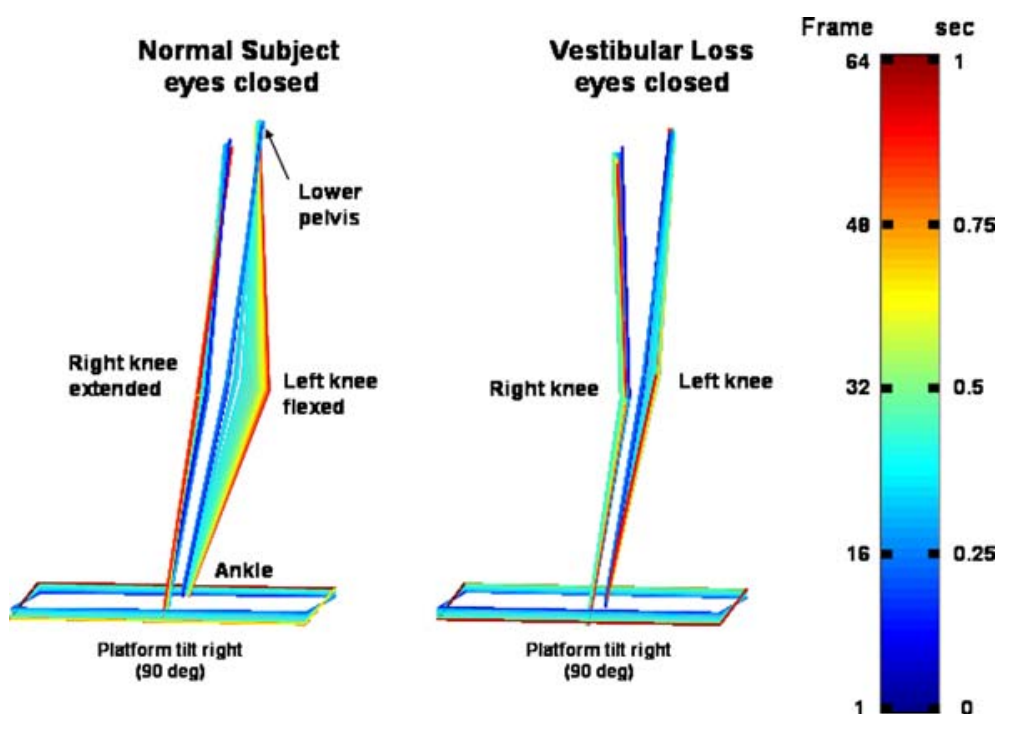

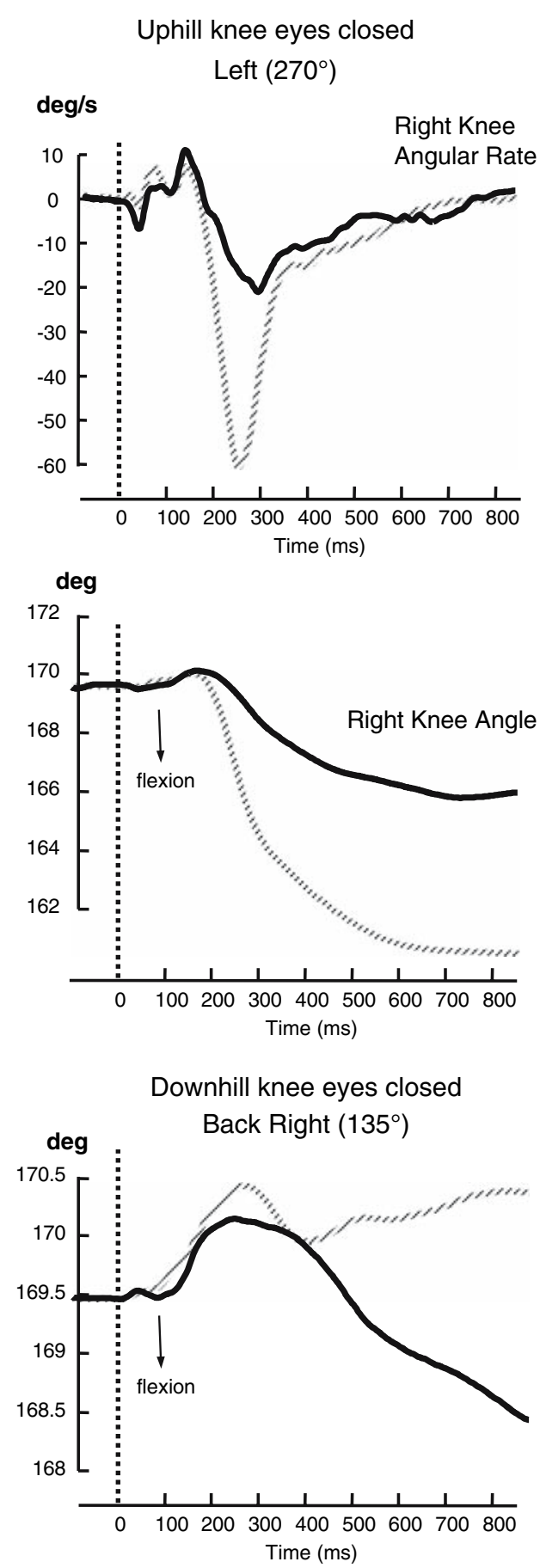

Fig. 10 Knee motion of VL and control subjects. On the left, the population average knee flexion angle and angular velocity under eyes closed conditions is shown by two sets of traces for the uphill (right) knee following a left-down tilt of the support-surface $\left(270^{\circ}\right)$ and for the downhill (right) knee for a 135 deg directed tilt. On the right bar diagrams illustrate the difference in uphill and downhill knee angular velocity and angles between the mean values of VL and control subjects

uphill knee-flexion velocity was influenced by perturbation direction in controls, ranging from $60 \%$ s for pure roll $\left(90^{\circ} \%\right.$ $\left.270^{\circ}\right), 40^{\circ} / \mathrm{s}$ for forward roll $\left(45^{\circ} / 315^{\circ}\right)$ and as little as $15^{\circ} / \mathrm{s}$
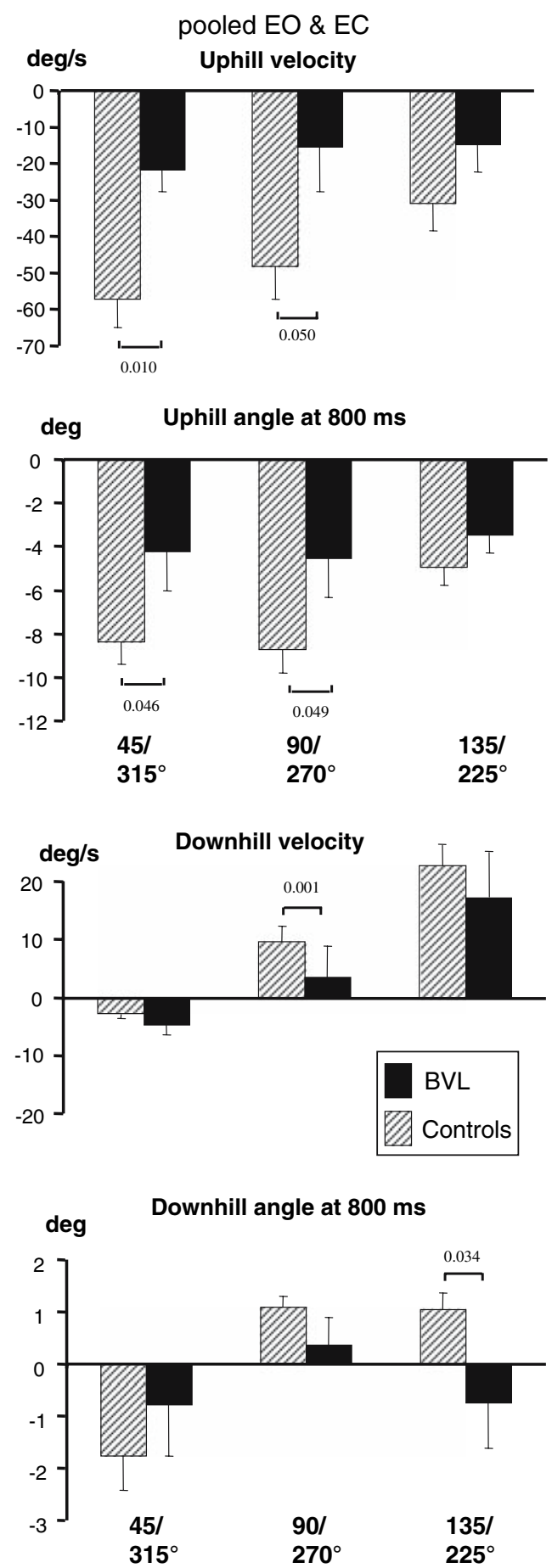

for roll perturbation directions pooled according to the pitch direction (for example, results for 45 and 315 perturbation directions are pooled) and visual condition (eyes open and closed) as no effect of vision was found. The velocity measurements were taken at the times of maximum differences (at ca. $250 \mathrm{~ms}$ ) and the angles at $800 \mathrm{~ms}$. Significant differences are marked by a horizontal bar and the $P$ value. Vertical bars on the columns indicated the size of the standard error of the mean (SEM)

for backward roll $\left(135^{\circ} / 225^{\circ}\right)$ perturbations. VL subjects had significantly less uphill knee angular velocity compared to controls (Fig. 10), with largest differences in peak 
velocities observed in forward roll (45/315), and roll (90/ $270)$ directions $(P<0.05)$. This difference in peak velocity measures was also reflected in differences in knee angle (Fig. 10). Flexion of the uphill knee (relative to platform tilt) was influenced by a significant main group effect at $300 \mathrm{~ms}\left(F_{(1,15)}=6.9, P<0.02\right)$, with VL subjects having significantly less knee flexion compared to controls across all perturbation directions. At $800 \mathrm{~ms}$, uphill knee flexion was found to be influenced by a significant group by direction interaction $\left(F_{(4,60)}=5.0, P<0.01\right)$. As shown in Fig. 10, VL subjects had significantly less flexion in the uphill knee at $800 \mathrm{~ms}$ for pure roll $\left(90^{\circ} / 270^{\circ}\right)$ and forward roll $\left(45^{\circ} / 315^{\circ}\right)$ directions $(P<0.05)$. There was no significant effect of group at 300 or $800 \mathrm{~ms}$ post-stimulus onset for flexion of the uphill hip joint.

Backwards and roll tilt requires an extension of the downhill leg for body stability. The peak angular velocity associated with extension of the downhill knee was significantly influenced by a group by direction interaction $\left(F_{(4,60)}=4.8, P<0.01\right)$, with VL subjects having significantly lower downhill knee velocity than controls for pure roll $\left(90^{\circ} / 270^{\circ}\right)$ directions $(P<0.05$, Fig. 10). Downhill knee movements were also significantly influenced by a significant group by direction interaction at $800 \mathrm{~ms}$ $\left(F_{(4,60)}=4.414, P<0.01\right)$ post-stimulus onset. As shown in Fig. 10, VL subjects compared to controls had a significantly more flexion instead of extension of the downhill knee during backward roll perturbations $\left(135^{\circ} / 225^{\circ}\right)$ at $800 \mathrm{~ms}(P<0.05)$. At $800 \mathrm{~ms}$ downhill hip angle was significantly influenced by a group by direction interaction $\left(F_{(4,60)}=3.3, P<0.02\right)$, with VL subjects using significantly less downhill hip extension compared to controls for forward roll $\left(45^{\circ} / 315^{\circ}\right)$ perturbations $(P<0.05)$.

\section{Arm displacements post $150 \mathrm{~ms}$}

As reported previously (Allum et al. 2002), both arms initially move toward the uphill side of the platform movement, resulting in abduction of the uphill arm and adduction of the downhill arm relative to the trunk (see also the stick of the control subject in Fig. 7). After $300 \mathrm{~ms}$ the arms returned to their initial position in controls but abducted more away from the body in VL subjects (see, for example, stick figures in Fig. 7 and downhill arm traces in Fig. 11). Significant interactions between group and direction were observed for arm displacements at $300 \mathrm{~ms}$ in both the uphill $\left(F_{(4,60)}=2.666\right.$, $P=0.041)$ and downhill $\left(F_{(4,60)}=2.721, P=0.038\right)$ arm. Post hoc comparisons revealed significant differences between VL subjects and controls in backward perturbations for the uphill arm $(P<0.05)$, and backward, backward roll and roll directions for the downhill arm $(P<0.05)$.

In summary, these biomechanical observations indicate that insufficient uphill leg flexion and downhill leg exten-

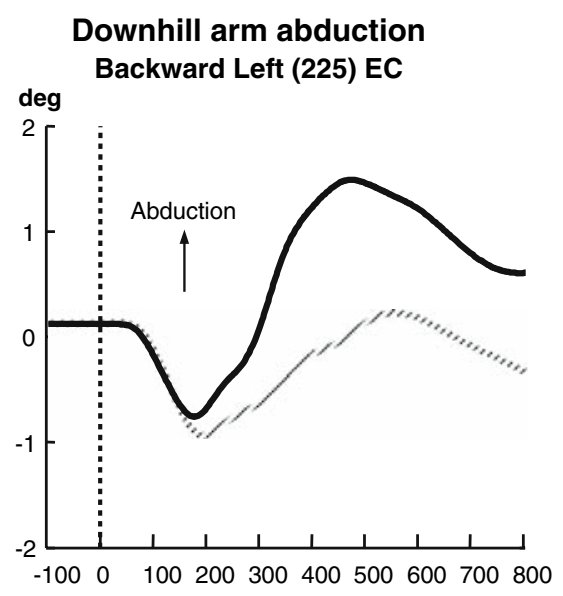

Downhill Arm Abduction at $\mathbf{3 0 0}$ ms (pooled EC\&EO)

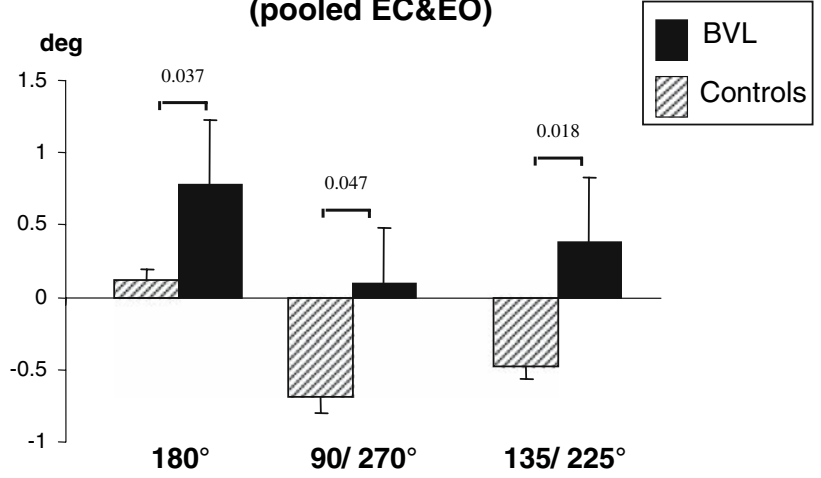

Fig. 11 Differences in arm abduction in VL and control subjects. The mean population traces for left arm abduction traces for a backward left $\left(225^{\circ}\right)$ tilt of the support surface under eyes closed conditions are shown. The column plots below show eyes closed means (and SEM) for $\mathrm{VL}$ and control subject

sion occurred in VL subjects for all forward pitch and roll perturbations $\left(45^{\circ}, 90^{\circ}, 270^{\circ}, 315^{\circ}\right)$ as well as the two backward roll directions $\left(135^{\circ}\right.$ and $\left.225^{\circ}\right)$. These differences, as well as those for arm abduction, were present at $300 \mathrm{~ms}$ well before instabilities were observed in trunk roll movements. No correlate in the knee movements was noted for the earlier instability in trunk pitch motion for the $180^{\circ}$ backward tilt direction.

Balance correcting muscle responses post $120 \mathrm{~ms}$

As reported previously (Carpenter et al. 2001) reduced amplitudes of EMG responses were observed in the soleus muscles of VL subjects compared to controls for forward directions, and increased amplitudes in paraspinal muscles for backward and ipsi-roll directions. New in this study is the observation that VL subjects had significantly lower amplitude balance correcting responses in deltoids compared to controls, with greater differences observed in EO than EC conditions. In contrast, no significant main effects 
of VL or interactions between group and direction were observed in the gluteus medius muscles.

\section{Discussion}

Vestibular loss affects roll tilt-induced leg flexion

This study examined the responses to support-surface tilt in different directions in vestibular loss subjects and controls. We demonstrated for the first time that the leg responses to roll tilt in humans mimic, to a large extent, the vestibularly driven responses of animals, that is with a leg flexion in the uphill leg and extension in the downhill leg. However, as shown in the schematic of Fig. 12, the polarity of vestibular inputs driving these responses must be different in humans compared to quadripeds because of the hinging of the trunk at the pelvis in humans in the direction opposite to tilt support-surface. We have demonstrated here that tilt of the head in humans follows that of the trunk. In cats, due to the biomechanics of quadripedal stance, both trunk and pelvis rotate in the direction of support-surface tilt (MacPherson et al. 2007). Assuming in the lack of evidence to the contrary, that head tilt also follows that of pelvis and trunk roll in quadripeds, then the direction of roll acceleration perceived by cats and humans for the same direction of tilt must be of different polarity (see Fig. 12). The uphill leg

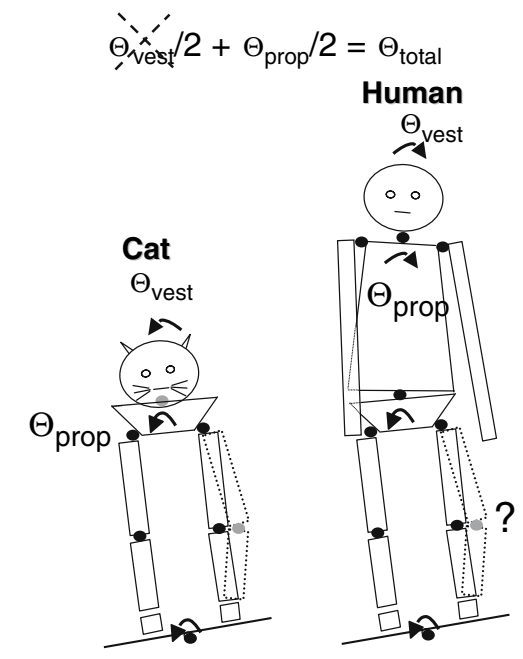

Fig. 12 Schematic illustrating the proposed misinterpretation of trunk position in space after vestibular loss in humans and cats. In human subjects a combination of vestibular signals due to head roll and proprioceptive signals from trunk roll in the opposite direction to support surface tilt provide information on the tilt of the body. In cats, the head is assumed to roll with the pelvis and trunk in the same direction as the support-surface tilt. It is assumed that the vestibular and proprioceptive signals are only correctly interpreted when both are present. With vestibular loss, the remaining proprioceptive signal is misinterpreted as less trunk roll and the compensating reaction of knee flexion is inadequate flexion response and downhill leg extension responses are, however, functionally similar. We will also argue below that because roll proprioception and vestibular information arrives simultaneously in humans the CNS probably processes this information simultaneously too with consequence that, if the vestibular component is lacking, vestibular loss (VL) subjects presumably assume less roll tilt has occurred, flex the uphill knee and extend the downhill knee inadequately and tend to fall in the direction of tilt.

Traditionally balance responses have received a different focus in two-legged humans and four-legged animals. Studies on the effect of vestibular loss in humans, have concentrated primarily on balance corrections that maintain pitch plane stability. Thus several authors have identified vestibular loss as cause for pitch plane instability in humans (Nashner et al. 1982; Keshner et al. 1987; Horak et al. 1990; Runge et al. 1998). Vestibular loss has been shown to lead to insufficient amplitudes of ankle muscle activity and excessive paraspinal muscle activity in VL subjects (Allum and Pfaltz 1985; Keshner et al. 1987; Allum and Honegger 1998; Carpenter et al. 2001), when the support surface is tilted backwards ( $180^{\circ}$ direction in our nomenclature). Balance control is then quite difficult for VL subjects and it is not easy for them to avoid falling over backwards, especially with eyes closed. We therefore were surprised to find in this study and in a previous study (Carpenter et al. 2001) even greater differences in trunk amplitudes between vestibular loss and normal subjects for roll tilts of the support surface. These tilts required the uphill knee to be flexed and the downhill knee extended as part of the compensatory response.

Numerous studies have examined the responses to roll tilts of the support surface in both normal and vestibular loss cats (Lindsay et al. 1976; Andersen et al. 1977; Peterson et al. 1980; Pompeiano 1984; Wilson and Schor 1999; MacPherson et al. 2007). It is generally accepted that vestibular-spinal inputs contribute significantly to muscle responses flexing the uphill hind- and fore-limbs and extending the downhill limbs (Krutki et al. 2003; Anderson et al. 1977; Pompeiano 1984). In these animal experiments, insufficient muscle activity following chronic loss of vestibular function was demonstrated (Wilson and Schor 1999; Pompeiano 1984). More recently MacPherson et al. (2007) have demonstrated that uphill leg muscle responses to tilt can be characterised by an overreaction not just a weaker response. Rather than executing less flexion of the uphill leg and extension of downhill leg as we have argued here for humans, these authors have argued that the responses to tilt are dominated by an active extension of the uphill leg pushing the animal downhill.

In VL subjects we observed the failure to flex the uphill knee adequately as early as $200 \mathrm{~ms}$ after stimulus onset in 
terms of knee flexion velocity (see Fig. 8) and later at $300 \mathrm{~ms}$ as inadequate knee flexion. This was well before instabilities were observed in trunk roll. Because the insufficient knee flexion was observed for all directions of tilt that lead to later trunk roll instabilities (forward roll and roll directions), we conclude that the insufficient knee flexion is the primary cause of the trunk instability. Instability in trunk roll was less for backward and roll tilts, but these directions required less knee flexion. However, the hip flexion of the uphill leg and extension of the downhill knee was also less in VL subjects for these directions. Our current results indicate that the VL subjects respond to the tilt with an uphill knee flexion and downhill knee extension (or even a failure to extend the downhill knee) only appropriate for a smaller amplitude of support surface roll tilt. The cause of the inadequate knee movements needs to be investigated. We assume that inadequate vestibular modulation of the knee flexion and extension muscles in both the uphill and downhill legs during balance corrections is the root cause of the roll instability in VL subjects. As only less flexion in the uphill leg is observed in humans, rather than extension, there is no evidence to suggest in humans a reversal of responses leading to an overreaction as has been suggested for cats (MacPherson et al. 2007). Furthermore our recordings to date in VL subjects have only revealed increased or decreased response amplitudes following vestibular loss (Allum and Pfaltz 1985; Allum and Honegger 1998; Carpenter et al. 2001) and not response reversals. Response reversals in quadriceps might, however, be consistent with different "wiring" of vestibulo-spinal reflexes, which takes into account the different direction of head roll movement following platform tilt.

In quadripeds, the lack of vestibular input has a similar effect on muscle responses in both fore limbs and hind limbs (Grillner and Hongo 1971; Wilson and Yoshida 1969). For this reason, biomechanically, a similar insufficient uphill-flexion and downhill forelimb extension would be expected in the arm muscles of humans. Due to the free arm movement, human arm responses have an entirely different function than those of the forelimbs of quadripeds. Firstly the arms are swung by the stimulus in the same direction as the initial trunk movement, uphill in young and middle aged (as the subjects in this study) adults (Allum et al. 2002). Therefore, the abducted uphill arm and adducted downhill arm immediately act, in the middle-aged adults we tested, as counterweight to the tendency for the COM to tip downhill. The downhill arm simply returns in normal subjects to its pre-stimulus positions (Fig. 9) and the uphill arm is slightly abducted after $300 \mathrm{~ms}$ as the trunk is stabilised with a constant lean uphill. In VL subjects the downhill arm was abducted more. We would propose that the downhill arm abduction due to changed amplitudes of responses in arm muscles is a secondary cause of trunk roll instability in vestibular loss subjects.
Timing of roll and pitch balance corrections

Previously we had noted that an earlier stimulus induced trunk roll compared to pitch was part of the biomechanical response of the body to the tilt perturbation on the support surface (Carpenter et al. 1999; Allum et al. 2003; Grüneberg et al. 2005). As the stimulus induced trunk roll motion occurs earlier than that of pitch, even when the stimulus is a combined roll and pitch tilt, we assumed that the balance control centres (BCC) presumably located in the CNS would require separate processing loops to generate balance corrections arresting unstable body motion in the roll and pitch planes (Carpenter et al. 2001). Furthermore, that roll balance corrections would be generated first as roll motion occurred first. In fact, balance corrections of body motion in the pitch and roll planes begin simultaneously with onsets of 90-120 ms across many body segments (Carpenter et al. 2001; Grüneberg et al. 2005). By shifting the onset of roll tilt with respect to the pitch tilt, it could be shown that roll tilt corrections do not begin earlier than those of pitch and that both roll and pitch commands appeared to be separately programmed (Grüneberg et al. 2005). Therefore it appears that BCC permit the roll instability of the trunk to continue well after the onset of roll movement in favour of generating roll and pitch balance correcting commands simultaneously across many segments. We argue that this may be another factor underlying the greater roll than pitch plane instability in VL subjects, in addition to how VL subjects may continue to incorrectly weight proprioceptive roll signals when vestibular signals are absent.

Simultaneous sensory processing times may account for greater roll instability with vestibular loss

Given the distributed nature of the proprioceptive inputs best related to the pitch and roll stimuli (pitch at the ankle, roll at the hip and lumbo-sacral joints) and the timing of vestibular responses to pitch and roll head accelerations, one possible explanation for the later and larger roll instability than pitch plane instability could be based on the different times when these sensory signals interact for perturbations in the roll and pitch directions. That is the types of processing BCC in the CNS use to form a perception of trunk roll and pitch may differ. If the head acceleration measurements we recorded represent the information transduced by the vestibular system to BCC, than it would appear from our results that stimulus related pitch directional and amplitude information is received by $\mathrm{BCC}$ in the CNS via vestibular afferents within $13 \mathrm{~ms}$ of stimulus onset whereas roll information is received some 20 ms later (see Figs. 1, 3).

Presumably the pitch rotation of the support-surface is transmitted as a vertical acceleration to the head with little delay because, along its longitudinal axis, the body is fairly 
rigid. In contrast, ankle proprioceptive inputs will reach the CNS much later. Taking the onset of the stretch reflex in ankle muscles as the time to reach the CNS (assuming the time taken for the reflex response to travel back to the muscle from the spinal cord is the same as that up to the CNS) this proprioceptive information will be present in the CNS at $46 \mathrm{~ms}$. The trunk will first start to pitch forward or backward some $50 \mathrm{~ms}$ later, leaving sufficient time to plan an appropriate balance-correcting response. The relative long delay, between the normally present vestibular input and proprioceptive input, suggests that alternative sources of vertical body acceleration signals could easily be integrated into the triggering and modulating proprioceptive signals from the ankle joint, if vestibular signals were absent. One such alternative signal might originate from load receptors in the spinal column registering the upward or downward thrust of the support-surface with pitch (Dietz et al. 1998).

Thus, we would argue that, in the pitch direction, proprioceptive and vestibular information is processed independently. This would allow VL subjects to use other proprioceptive information instead of vestibular information to successfully maintain balance in pitch. Nonetheless, the use of alternative proprioceptive inputs, in the event of vestibular loss is, as we have demonstrated, still deficient. Poor pitch balance control remains, possibly because VL subjects tend to weight ankle proprioceptive inputs more heavily (Peterka and Loughlin 2004). The result would be a greater orientation of the body to the support surface tilt rather than to the vertical.

Roll proprioceptive information arrives at the CNS almost simultaneously with vestibular roll information. This is because roll plane perturbations elicit head movements later than pitch perturbations (Figs. 1, 3). Roll accelerations of the head, according to our measurements, commence around $31 \mathrm{~ms}$. At the same time roll tilt is transmitted to the pelvis, with the legs acting as a parallelogram. Thereby, rotation of the pelvis with respect to the legs and trunk occurs leading to stretch and unloading responses in bilateral gluteus medius and paraspinal muscles at $28 \mathrm{~ms}$. Thus the time difference between the arrival vestibular and proprioceptive roll sensory inputs is much shorter than that for pitch inputs. The small difference in the case of roll may lead to a restricted choice of alternative sensory signals for roll motion, especially if the CNS programs roll directed balance corrections based on a sensory transformation, similar to that proposed by Mergner (1997). Based on this proposal, the sensory information can only be interpreted when the sensory signals are combined. Furthermore, the larger variance in roll and lateral linear head accelerations and the changed relationship to stimulus roll (Fig. 4) in BVL subjects may preclude the use of cervical collic proprioceptive inputs as an alternative to the absent vestibular signal. As illustrated in Fig. 12 less of the vestibular information (and the failure to use a replacement signal with a similar timing and amplitude relationship) will lead to an inadequate estimate of counter-tilt trunk lean in humans and pelvis and trunk tilt in quadripeds labelled $\theta$ total in Fig. 12. The trunk lean in humans, as we have previously shown, is related to the amount of support surface roll tilt (Carpenter et al. 1999). The result will be a misinterpretation of the perturbation amplitude (as is shown in Fig. 12, for the simple case in which each input is weighted by 0.5 ). This will lead to insufficient knee flexion to correct the response and movement of the trunk to the side of tilt. It has been suggested that a similar misinterpretation process occurred in VL cats during voluntary horizontal head turns Stapley et al. (2006) or following support-surface tilt (MacPherson et al. 2007). Furthermore, spinal-cerebellar ataxia patients with normal vestibulo-ocular reflexes also provide insufficient knee flexion on roll tilt (Bakker et al. 2006). This latter finding would support the viewpoint that a sensory integration deficit rather than loss of a sensory signal alone is the cause of balance instability in roll.

It should be noted that our conclusions on the perception of head accelerations by vestibular sensory systems are based on direct measurements of head accelerations recorded with high sample rates $(1 \mathrm{kHz})$. Interpretations of head accelerations based on double differentiation of hand position signals with low sampling rates $(\mathrm{ca} .100 \mathrm{~Hz}$ ) of position signals will be limited by both the low sampling rate and inherent noise following differentiation of position signals (see for example Forssberg and Hirschfeld 1994 or MacPherson et al. 2007). For this reason, early work on postural control in the pitch plane focussed more on proprioceptive rather than on vestibular sensory inputs contributing to balance corrections (Nashner et al. 1982; Diener et al. 1988). Direct measurements of head accelerations in the pitch plane provided a correction to this focus (Allum and Pfaltz 1985; Runge et al. 1998).

It is possible that our use of foot straps and heel stops to keep subjects in the same position on the platform with respect to its axes of rotation throughout the experiments has the advantage of leading to consistent head acceleration responses with small variance. This disadvantage is that, by adding additional somatosensory inputs at the feet, balance correcting responses may be changed. Based on the similarity pitch plane responses for rotation and translation of the support-surface recorded in our work (Allum and Pfaltz 1985; Allum and Honegger 1998; Bloem et al. 2000) and that of others who did not use foot straps (Runge et al. 1998; Horak et al. 1990; Diener et al. 1988) we conclude that responses in the pitch plane are not altered by presence of the foot straps. For the roll plane, comparisons with the work of other authors are not available. Thus we cannot currently exclude an alteration of roll plane responses due to the foot straps and heel stops. 
In summary we have observed greater roll than pitch instability following vestibular loss and attempted to link this directional deficit to differences in the processing of proprioceptive and vestibular signals based on the time when signals might be processed in the CNS in order to form balance corrections. We have developed a concept of sensory interaction based on vestibular and proprioceptive signals being processed separately in time for pitch perturbation directions but in a combined manner for roll. Presumably a greater adaptation by balance control centres is required for roll perturbations as a reprogramming takes place with alternative sensory signals, a task the CNS appears not to cope with as adequately in the roll as in the pitch direction, because the combined processing mode in roll leads to misinterpretation of roll stimulus amplitude. The net result is a change in the vestibular modulation of the amplitudes of uphill knee flexion and downhill knee extension required to correct centre of mass tilt induced by the support-surface roll tilt. We assume the amplitudes of knee flexor and extensor muscle activity during early balance corrections are inadequate in VL subjects.

Acknowledgments This work was supported by National Swiss Research Fund grant number 3100A0-104212 to JHJ Allum. Funding for MG Carpenter was provided by the Natural Sciences and Engineering Research Council of Canada.

\section{References}

Anderson JH, Soechting JF, Terzuolo CA (1977) Dynamic relations between natural vestibular inputs and activity of forelimb extensor muscles in the decerebrate cat. I. Motor output during sinusoidal linear accelerations. Brain Res 120:1-15

Allum JHJ, Pfaltz CR (1985) Visual and vestibular contributions to pitch sway stabilization in the ankle muscles of normals and patients with bilateral peripheral vestibular deficits. Exp Brain Res 58:82-90

Allum JHJ, Honegger F, Schicks H (1993) Vestibular and proprioceptive modulation of postural synergies in normal subjects. J Vest Res 3:59-85

Allum JHJ, Honegger F, Acuña H (1995) Differential control of leg and trunk muscle activity by vestibulo-spinal and proprioceptive signals during human balance corrections. Acta Otol Laryngol (Stockh) 115:124-129

Allum JHJ, Honegger F (1998) Interactions between vestibular and proprioceptive signals in triggering and modulating human balance-correcting responses differ across muscles. Exp Brain Res 121:478-494

Allum JHJ, Ledin T (1999) Recovery of vestibulo-ocular function in subjects with acute peripheral vestibular loss. J Vest Res 9:135144

Allum JHJ, Carpenter MG, Bloem BR, Honegger F, Adkin AL (2002) Age-dependent variations in the directional sensitivity of balance corrections. J Physiol (Lond) 542:643-663

Allum JHJ, Carpenter MG, Honegger F (2003) Directional aspects of balance corrections in man. IEEE Eng Med Biol Mag 22:37-47

Bakker M, Allum JHJ, Visser JE, Grüneberg C, Van de Warrenburg BPC, Kremer BH, Bloem BR (2006) Postural responses to multidirectional stance perturbations in cerebellar ataxia. Exp Neurol 202:21-35
Bloem BR, Allum JHJ, Carpenter MG, Honegger F (2000) Is lower leg proprioception essential for triggering human balance corrections? Exp Brain Res 130:375-391

Bloem BR, Allum JHJ, Carpenter MG (2002) Triggering of balance corrections and compensatory strategies in a patient with total leg proprioceptive loss. Exp Brain Res 142:91-107

Carpenter MG, Allum JHJ, Honegger F (1999) Directional sensitivities of stretch reflexes and balance corrections for normal subjects in the roll and pitch planes. Exp Brain Res 129:93-113

Carpenter MG, Allum JHJ, Honegger F (2001) Vestibular influences on human postural control in combination of pitch and roll planes reveal differences in spatio temporal processing. Exp Brain Res 140:95-111

Carpenter MG, Allum JHJ, Honegger F, Adkin AL, Bloem BR (2004) Postural abnormalities to multidirectional stance perturbations in Parkinson's disease. J Neurol Neurosurg Psychiat 75:1245-1254

Diener HC, Horak FB, Nashner LM (1988) Influence of stimulus parameters on human postural responses. J Neurophysiol 59:1888-1905

Dietz V (1998) Evidence for a load receptor contribution to the control of posture and locomotion. Neurosci Biobehav Rev 22:495-499

Forssberg H, Hirschfeld H (1994) Postural adjustments in sitting humans following external perturbations. Exp Brain Res 97:515527

Grillner S, Hongo T (1972) Vestibulo spinal effects on motoneurons and interneurons in the lumbosacral cord. Prog Brain Res 37:243262

Grillner S, Hongo T, Lund S (1971) Convergent effects on $\alpha$-motoneurons from the vestibulospinal tract and a pathway descending in the medial longitudinal fasciculus. Exp Brain Res 12:457-479

Grüneberg C, Allum JHJ, Honegger F, Bloem BR (2004) The influence of artificially increased hip and trunk stiffness on balance control in the pitch and roll planes. Exp Brain Res 157:472-485

Grüneberg C, Duysens J, Honegger F, Allum JHJ (2005) Spatio-temporal separation of roll and pitch balance correcting commands in man. J Neurophysiol 94:3143-3158

Henry SM, Fung J, Horak FB (1998) EMG responses to maintain stance during multidirectional surface translations. J Neurophysiol 80:1939-1950

Horak FB, Nashner LM, Diener HC (1990) Postural strategies associated with somatosensory and vestibular-loss. Exp Brain Res $82: 167-177$

Inglis JT, Macpherson JM (1995) Bilateral labyrinthectomy in the cat: effects on the postural response to translation. J Neurophysiol 73:1181-1191

Katz R, Pierrot-Deseilligny E (1999) Recurrent inhibition in humans. Prog Neurobiol 57:325-355

Keshner EA, Allum JHJ, Pfaltz CR (1987) Postural coactivation and adaptation in the sway stabilizing responses of normals and patients with bilateral vestibular deficit. Exp Brain Res 69:77-92

Krutki P, Jankowska E, Edgley SA (2003) Are crossed actions of reticulospinal and vestibulospinal neurons on feline motoneurons mediated by the same or separate commissural neurons? J Neurosci 23:8041-8050

Lacour M, Xerri C, Hugen M (1979) Compensation of postural reactions to fall in the vestibular neurectomized monkey. Role of remaining labyrinthic afferences. Exp Brain Res 37:563-580

Lindsay KW, Roberts TD, Rosenberg JR (1976) Asymmetric tonic labyrinth reflexes in the decerebrate cat. J Physiol 261:583-601

MacPherson JM (1988) Strategies that simplify the control of quadrupedal stance and forces at the ground. J Neurophysiol 60:204-217

MacPherson JM, Everaert DG, Stapley PJ, Ting LH (2007) Bilateral vestibular loss in cats leads to active destabilization of balance during pitch and roll rotations of the support-surface. J Neurophysiol 97:4357-4367 
Mergner T, Huber W, Becker W (1997) Vestibular-neck interaction and transformation of sensory coordinates. J Ves Res 7:347-367

Nashner LM, Black FO, Wall C III (1982) Adaptation to altered support and visual conditions during stance in patients with vestibular deficits. J Neurose 5:536-544

Perry SD, McIlroy WE, Maki BE (2000) The role of plantar cutaneous mechanoreceptors in the control of compensatory stepping reactions evoked by unpredictable, multi-directional perturbation. Brain Res 877:401-406

Peterka RJ, Loughlin PJ (2004) Dynamic regulation of sensorimotor integration in human postural control. J Neurophysiol 91:410 423

Peterson BW, Fukushima K, Hirgi N, Schor RH, Wilson VJ (1980) Responses of vestibulospinal and recticulospinal neurons to sinusoidal vestibular stimulation. J Neurophysiol 43:1236-1250

Pompeiano O (1984) Excitatory and inhibitory influences on the spinal cord during vestibular and neck reflexes. Acta Otolaryngol (Stockh) Suppl 406:5-9

Roberts TMD (1995) Understanding balance. The mechanics of posture and locomotion. Chapman and Hall, London, UK
Runge CF, Shepert CL, Horak FB, Zajac FE (1998) Role of vestibular information in initiation of rapid postural responses. Exp Brain Res 122:403-412

Stapley PJ, Ting LH, Kuifu C, Everaert DG, MacPherson JM (2006) Bilateral vestibular loss leads to active destabilization of balance during voluntary head turns in the standing cat. J Neurophysiol 95:3783-3797

Ting LH, MacPherson JM (2004) Ratio of shear to ground-reaction force may underlie the directional tuning of the automatic postural response to rotation and translation. J Neurophysiol 92:808-823

Wilson VJ, Yoshida M (1969) Comparison of effects of stimulation of Dieters' nucleus and medial longitudinal fasciculus on neck, forelimb, and hindlimb motoneurons. J Neurophysiol 32:743-758

Wilson VJ, Melvill Jones G (1979) Mammation vestibular physiology. Plenum, New York, pp 239-245

Wilson VJ, Schor RH, Suzuki I, Parks BR (1986) Spatial organisation of neck and vestibular reflexes acting on the forelimbs of the decerebrate cat. J Neurophysiol 55:514-526

Wilson VJ, Schor RH (1999) The neural substrate of the vestibulo colic reflex. What needs to be learned. Exp Brain Res 129:483-493 